\title{
Simulation of radiation damping in rings using stepwise ray-tracing methods
}

\author{
F. Méot
}

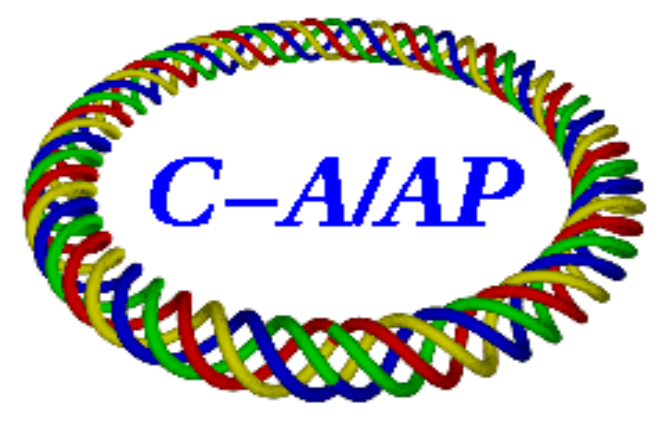

\section{Collider-Accelerator Department Brookhaven National Laboratory Upton, NY 11973}

Notice: This document has been authorized by employees of Brookhaven Science Associates, LLC under Contract No. DE-AC02-98CH10886 with the U.S. Department of Energy. The United States Government retains a nonexclusive, paid-up, irrevocable, world-wide license to publish or reproduce the published form of this document, or allow others to do so, for United States Government purposes. 


\title{
Simulation of radiation damping in rings using stepwise ray-tracing methods
}

\author{
F. Méot \\ (fmeot@bnl.gov)
}

BNL C-AD, Upton, LI, NY 11973

\begin{abstract}
The ray-tracing code Zgoubi has been subject to benchmarking regarding synchrotron radiation damping in rings, in the past months. This work is reported here. It has been performed in view in particular of further spin diffusion simulations benchmarking, and application to electron beam polarization transmission in e-RHIC.
\end{abstract}




\section{Contents}

1 Introduction

2 Ray-tracing and SR methods 3

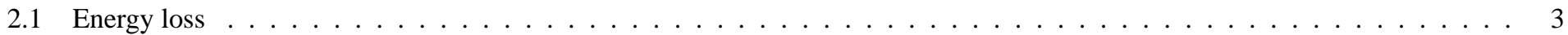

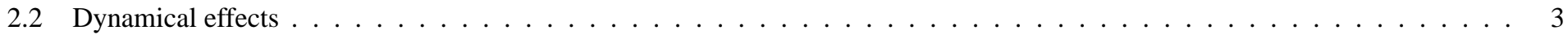

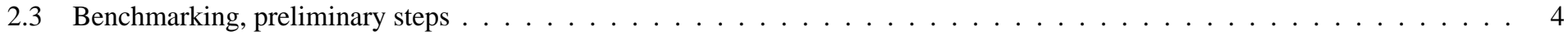

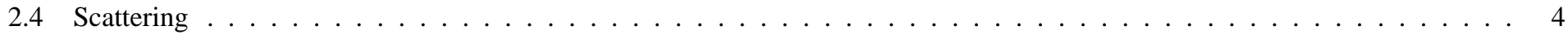

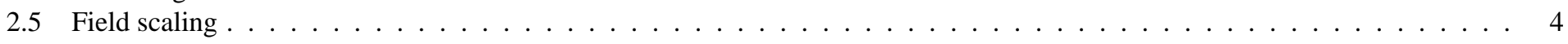

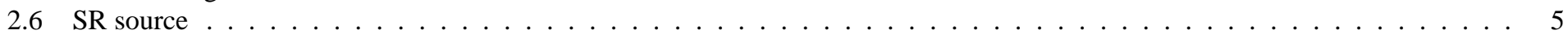

3 Chasman-Green test lattice 5

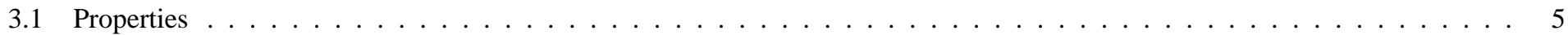

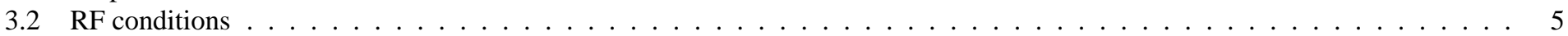

4 Benchmarking method $\quad 6$

5 Damping simulations $\quad 8$

6 Coupled motion 13

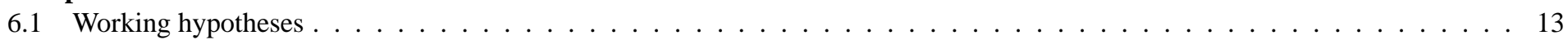

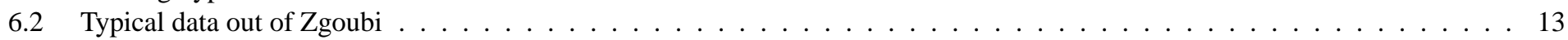

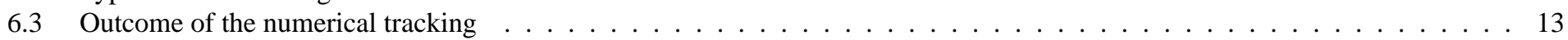

7 CPU time considerations $\quad 14$

8 Comments / conclusions $\quad 14$

Appendix

A SR in Zgoubi, earlier benchmarking 15

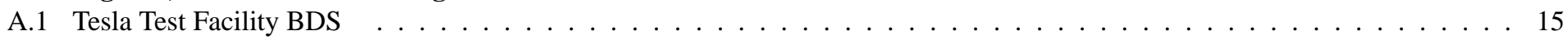

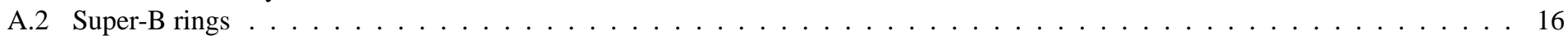

$\begin{array}{lll}\text { B Zgoubi method } & 17\end{array}$

$\begin{array}{lrr}\text { C Simulation of energy loss } & 17\end{array}$

D Chasman-Green lattice parameters, out of Zgoubi 18

E Typical Zgoubi i/o files

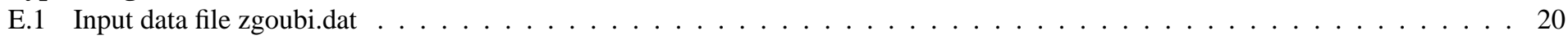

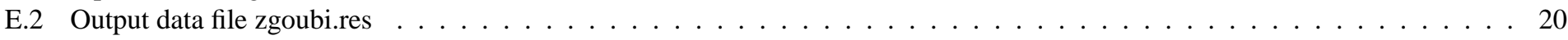

F Tracking, typical data $\quad \ldots 22$

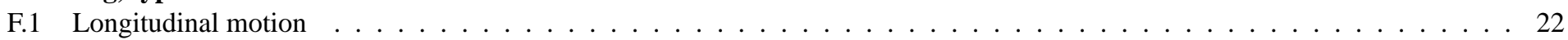

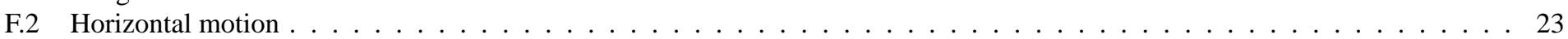

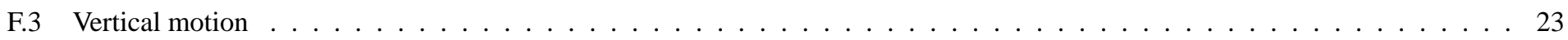

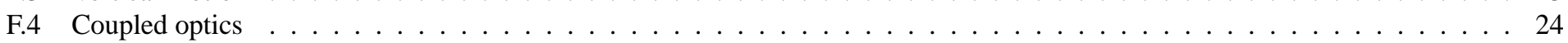

\begin{tabular}{ll} 
References & 25 \\
\hline
\end{tabular} 


\section{Introduction}

The ray-tracing code Zgoubi [1] has recently been subject to benchmarking regarding synchrotron radiation (SR) damping in rings. This work is reported here.

Stochastic SR was first introduced in Zgoubi for assessing emittance perturbation in the beam delivery system of the "Tesla Test Facility" (an early, European, test version of the "Linear Collider"), see App. A.1, or Ref. [2] for details. These developments were based on methods implemented earlier in the DYNAC dynamics code developed at Saclay [3] as a design tool for the recirculating arcs in the "ALS" and "ELFE" electron recirculator projects $[4,5]$. However, although presumably straightforwardly operational in rings, since ray-tracing in rings does not differ from ray-tracing in beam lines, the correctness of damping effects still had to be checked ${ }^{1}$.

This work is motivated by on-going activities in the field of high energy electron machines and other recirculators, whereas Zgoubi in addition allows spin tracking [8] which had motivated its use in design studies regarding the $\mathrm{e}^{+}-\mathrm{e}^{-}$asymmetric collider project, super-B [9], see App. A.2. It is planned, in the future, to benchmark SR effects on spin dynamics, as spin diffusion, Sokholov-Ternov radiative polarization. Although spin dynamics is not addressed here, the present work represents a preliminary stage in that direction. A goal at present is to have Zgoubi available for spin diffusion studies regarding the eRHIC project at BNL [10].

All Zgoubi input and output files that have been run and used in relation with this benchmarking work have been archived in the Zgoubi repository area on the C-AD network, at

\section{/rap/lattice_tools/zgoubi/library/SRLoss/rings_benchmarking}

guidelines can be found there in a dedicated "README" file. Any "zgoubi.dat" file ${ }^{2}$ therein can be copied and re-run, using the executable /rap/lattice_tools/zgoubi/zgoubi

Detailed indications on that are given in due place all along the present report.

\section{Ray-tracing and SR methods}

The ray-tracing code Zgoubi calculates particle trajectories in arbitrary static fields by stepwise integration of the Lorentz equation of motion, using a method of truncated Taylor series (see App. B), which provides high accuracy, and relatively fast, stepwise computation (see section 7).

\subsection{Energy loss}

Given a particle travelling in the magnetic field of an arbitrary optical element or field map, Zgoubi may compute, upon user's request, the energy loss due to stochastic photon emission, and its effect on the particle motion, at each integration step. The energy loss is calculated in a classical manner, based on the two random processes, namely,

- probability of emission of a photon over an integration step,

- energy of the emitted photon.

The effects on particle dynamics are either limited to the alteration of energy, or may include scattering (namely, change in the direction of the momentum vector due to the angle of emission of the photon with respect to particle velocity vector), following user's requests regarding working conditions. Possible scattering involves a third random process,

- the photon emission angle,

otherwise assumed null.

Main aspects of the method have been detailed in Ref. [2], an outline is given in App. C.

\subsection{Dynamical effects}

Energy kick The energy of a particle is updated after each integration step $\Delta s$. It is obtained by summation of the individual energies (Eq. 14, p. 17) of the $k$ photons (Eq. 12, p. 17) emitted along $\Delta s$.

SR statistics in uniform field will therefore converge towards the following averages :

- energy loss ${ }^{3}$ by a particle in a step $\Delta s=\rho \Delta \theta$

$$
\Delta E=2 r_{0} E_{0} \gamma^{4} \frac{\Delta \theta}{3 \rho}=\frac{2}{3} r_{0} c \gamma^{3} B \Delta \theta
$$

with $B=$ rigidity $/ \rho$ the local field value, $E_{0}=m_{0} c^{2}$ the particle rest energy, $\gamma=E / E_{0}, r_{0}=e^{2} / 4 \pi \epsilon_{0} m_{0} c^{2} \approx 2.8179403210^{-15} \mathrm{~m}$ the classical radius of the electron,

\footnotetext{
${ }^{1}$ Note also, for completeness although in a different area, that Zgoubi provides as well SR spectrum computation in arbitrary magnetic fields, and was used for the assessment of a mini-wiggler based profile monitor at LEP [6] and for the design of the LHC undulator-radiation based profile monitors [7].

${ }^{2}$ Zgoubi requires a single input data file, "zgoubi.dat", and will deliver outputs in, a minima, "zgoubi.res" result file, and when explicitly requested, various other files as "zgoubi.fai", a record of turn by turn informations as particle coordinates and spin, "zgoubi.plt", a record of coordinates and fields inside optical elements, see the Users' Guide for details [1].

${ }^{3}$ An other form of the familiar relation $\Delta E / E \approx 1.878610^{-15} \gamma^{3} \Delta \theta / \rho$.
} 
- induced beam energy spread ${ }^{4}$

$$
\sigma_{\Delta E / E}=\frac{\sqrt{110 \sqrt{3} \hbar c / \pi \epsilon_{0}}}{576 E_{0}^{2}} \gamma^{5 / 2} \frac{\sqrt{\Delta \theta}}{\rho}
$$

\subsection{Benchmarking, preliminary steps}

At this stage, the installation of the Monte Carlo simulation in Zgoubi can be benchmarked for these quantities, using, following the hypotheses in Sec. 3, $\rho=24.95549 \mathrm{~m}$, iso-magnetic lattice. From a practical point of view, in order to stick to ideal theoretical conditions, a single bend is tracked once-through (so to avoid such effects as orbit spiraling, momentum spread, that may be sensible over a large ring or in presence of RF compensation).

Results are given in Tab. 1. The classical, theoretical formulæ used are recalled in the rightmost column. $E_{s}$ is the total energy of the synchronous particle, $\mathcal{C}_{\gamma}=\frac{4 \pi}{3} \frac{r_{0}}{\left(m_{0} c^{2}\right)^{3}} \approx 8.84627610^{-5}\left[\mathrm{~m} / \mathrm{GeV}^{3}\right], m_{0}=0.510998928 \mathrm{MeV}$. The values so computed are for $E_{s}=6 \mathrm{GeV}$, they are converged numerically, up to the last digit shown in the table, in terms of the integration step size in the bend $(\Delta s=1 \mathrm{~cm}$ in Eq. 10 , App. B) and of the number of radiated photons (made large enough, via the number of passes), this is shown in Fig. $1:$ the various trials displayed (various random seeds, various integration step size in the bend) show that, for a 3000 particle batch, both the energy loss $U_{s}$ and the critical energy $\epsilon_{c}$ converge to their asymptotic values in a few once-through passes across the dipole.

It can be seen that the agreement between Zgoubi tracking data (col. 3 in Tab. 1) and theoretical expectations (col. 4) is very good, this is considered to validate the SR Monte Carlo installation in Zgoubi.

Additional details regarding SR data that Zgoubi generates and records when ray-tracing in bends, including infos regarding the data in Tab. 1, can be found under the item "BEND" in App. E.2. Monte Carlo losses at higher energies are further shown to yield as good agreement with Eq. 1, in Tab. 8, page 12.

Table 1: Preliminary benchmarking : SR loss characteristics in the Chasman-Green cell defined in Tab. 2. These quantities have been computed from a large number of once-through passes, of a 3000 particle batch, in a single dipole, they are shown scaled to a full turn (64 such dipoles).

\begin{tabular}{lcccl}
\hline $6 \mathrm{GeV}$ & Units & Zgoubi & \multicolumn{2}{c}{ Theoretical } \\
tracking & value & formula \\
\hline Energy loss, $U_{s}$ & $\mathrm{MeV} /$ turn & 4.59565 & $4.59565=\frac{\mathcal{C}_{\gamma}}{2 \pi} E_{s}^{4} \oint \frac{d s}{\rho(s)^{2} \stackrel{i s o-\rho}{=} \mathcal{C}_{\gamma} \frac{E_{s}^{4}}{\rho}}$ \\
Critical photon energy, $\epsilon_{c}$ & $\mathrm{keV}$ & 19.2051 & $19.2051=\frac{3 \bar{h} \gamma^{3} c}{2 \rho e}$ \\
Nb. of average photons & /turn/particle & 777.12 & $777.12=\frac{U_{s}}{\bar{\epsilon}}$ \\
\hline
\end{tabular}

\subsection{Scattering}

Trajectory scattering may assume for simplicity a cylindrical-symmetric Gaussian distribution

$$
p(\xi)=\exp \left(-\frac{\xi^{2}}{2 \sigma_{\xi}^{2}}\right)
$$

of the photon emission angle $\xi$ with respect to the particle velocity. For simplicity as well $\sigma_{\xi}$ may be considered independent of photon energy $\epsilon$, with value $\approx 1 / \gamma$. Whether these two approximations hold may be problem dependent, however these hypotheses may easily be improved if this is found necessary.

Accounting for scattering is an option in Zgoubi. Since its effect on beam divergence is very small in the present benchmarking conditions (mainly a matter of asymptotic vertical invariant value), it does not need be (and is not) taken into account.

\subsection{Field scaling}

Particle stiffness decrease upon SR loss entails perturbation of focusing properties due to increased strength of the magnets.

In the case of single-pass beam lines, this effect may be taken care of upon user's request, resulting in the scaling of magnetic fields to the theoretical average energy loss, namely (Eq. 1)

$$
\Delta E_{\text {scaling }}[e V]=\sum_{\text {bends }} \frac{2}{3} r_{0} c \gamma^{3} B \Delta \theta
$$

Note the following: (i) using that analytical expression in computing the scaling coefficient is preferred to averaging the energy loss from the tracked particle population, since in the latter case it would make it dependent on the accuracy of the statistics ; (ii) doing so the contribution

\footnotetext{
${ }^{4}$ That takes the form $\sigma_{\Delta E / E}=3.79410^{-14} \gamma^{5 / 2} \sqrt{\Delta \theta} / \rho$ for electrons.
} 


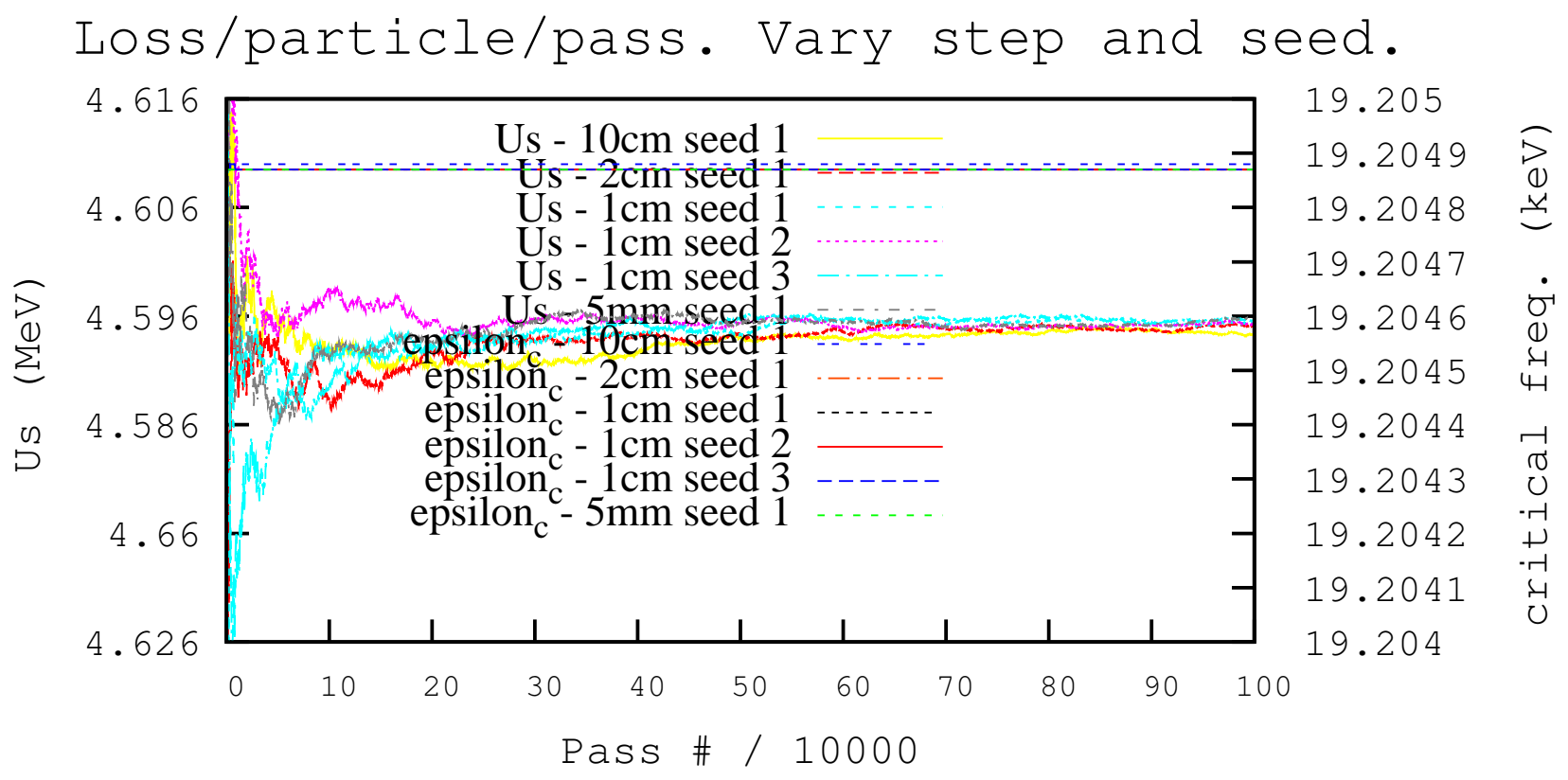

Figure 1: Convergence (running average) of the energy loss towards $4.5956 \mathrm{MeV} /$ turn (lower curve bundle, left vertical scale), and of the critical frequency towards $19.20 \mathrm{keV}$ (top curve, right vertical scale), as a function the number of passes of a 3000 particle batch, through the $2.45 \mathrm{~m}$ dipole, at $6 \mathrm{GeV}$.

of other magnets (quadrupoles, sextupoles, etc.) is not accounted for in the scaling, in the present state of Zgoubi coding, however this can easily be changed.

In storage rings, bends and lenses are normally operated at fixed field, whereas the RF takes care of restoring the energy lost, hence the damping effects. This is the subject of the present benchmarking study and will be discussed in detail. In pulsed regime as in booster injectors, the same process of energy recovery by the RF holds, thus requiring no particular measures on Zgoubi simulations side, save ad hoc synchronous RF phase considerations.

\subsection{SR source}

Upon user's request, SR loss can be limited to particular classes of optical elements, for instance bends alone, or bend + quadrupole magnets, etc.

This is so, for the flexibility of design studies, beam and spin dynamics studies, and other benchmarking purposes.

\section{Chasman-Green test lattice}

\subsection{Properties}

A Chasman-Green cell (aka double-bend achromat, DBA) is considered in the present benchmarking study, for the reason that a number of quantities relevant to beam dynamics under SR effects can be derived analytically in that case, as the chromatic invariant $\mathcal{H}$, equilibrium emittances, damping times, etc.

A variant of the ESRF super-cell (a double-DBA) is used, a storage ring is built from 16 such super-cells, whereas various storage energies will be considered, taken in the range $6 \mathrm{GeV}$ (actual ESRF energy) to $18 \mathrm{GeV}$.

Tab. 2 gives the general optical parameters of the lattice and ring, the optical functions are displayed in Fig. 2.

\subsection{RF conditions}

SR losses in bends over the ring circumference amount to

$$
U_{s}=\frac{\mathcal{C}_{\gamma}}{2 \pi} \beta^{3} E_{s}^{4} I_{2} \approx \frac{\mathcal{C}_{\gamma}}{2 \pi} E_{s}^{4} I_{2} \stackrel{i s o-\rho}{=} \mathcal{C}_{\gamma} \frac{E_{s}^{4}}{\rho} \quad \text { or, ultra-relativistic electrons } \quad U_{s}[k e V / \text { turn }] \approx 88.463 \frac{E_{s}^{4}[\mathrm{GeV}]}{\rho[m]}
$$

yielding for instance

$$
U_{s} \approx 4.6 \mathrm{MeV} / \text { turn - } 2.45 \mathrm{MW} \text { power - in the ESRF }
$$

Numerical values, for $6 \mathrm{GeV}$ electrons, are given in Tab. 1. The radiated energy is restored by the RF system. A single cavity is accounted for in the present simulations, with parameters as listed in Tab. 3. 


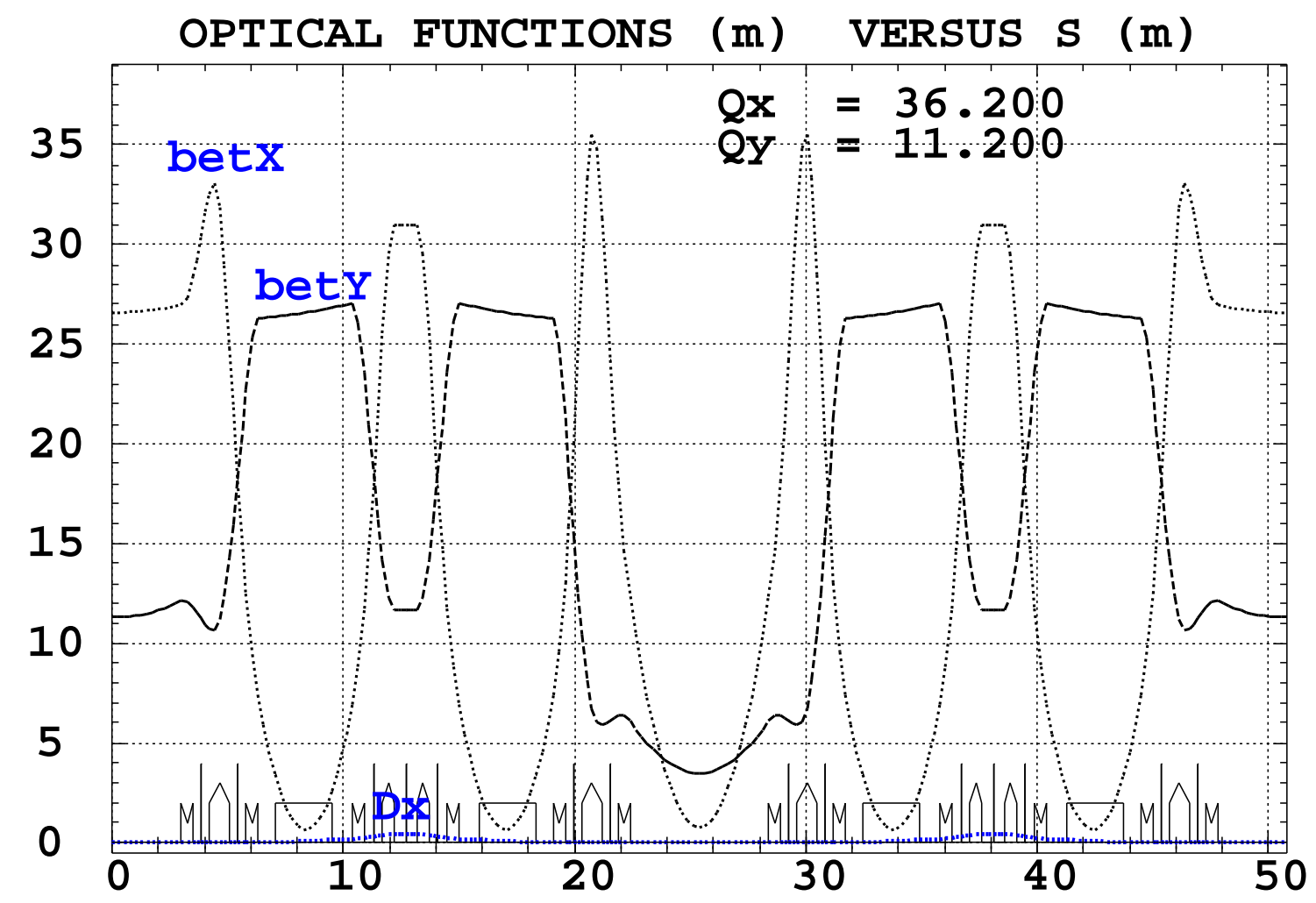

Figure 2: Optical functions in ESRF type Chasman-Green super-cell.

\section{Benchmarking method}

Benchmarking is based on monitoring the only thing Zgoubi can produce : particle coordinates, versus time or turn number. They are dealt with as follows.

The transverse and longitudinal motion invariants in the absence of perturbation can be written [11]

$$
\begin{cases}\epsilon_{z}=\left[z^{2}+\left(\alpha_{z}(s) z+\beta_{z}(s) z^{\prime}\right)\right] / \beta_{z} & z=x \text { or } y, \text { transverse } \\ \epsilon_{l}=(\Delta E)^{2}=(\Delta E)^{2}+\frac{1}{\Omega_{s}^{2}}\left(\frac{d \Delta E}{d t}\right)^{2} & \text { longitudinal }\end{cases}
$$

Under the effect of SR, individual invariants cannot be determined, averages over particle ensembles are considered instead, they evolve according to

towards

$$
\frac{d \bar{\epsilon}}{d t}=-\frac{\bar{\epsilon}}{\tau}+C \quad[\overline{(*)}=\text { average over particles }]
$$

with damping time

$$
\bar{\epsilon}_{\text {equil. }}=C \tau
$$

$$
\tau=\frac{T_{r e v} E_{s}}{U_{s} J_{x, y, l}}
$$

with $J_{x, y, l}$ the partition numbers, respectively horizontal, vertical, longitudinal, which, in passing, satisfy

$$
J_{x}+J_{y}+J_{l}=4, \quad \text { with in particular } J_{y}=1 \text { in a planar ring. }
$$

We will in general omit the average bar over $\epsilon_{x, y, l}$ in the rest of the paper, as long as there is no ambiguity.

\section{An example of Zgoubi outcomes, regarding these theoretical quantities :}

Vertical damping, case of a single particle with $6 \mathrm{GeV}$ energy, and 20000 turn tracking in the 16-cell ring, tracking results are summarized in Fig. 3.

The damping envelopes in the left plot satisfy

$$
\pm e_{y}(t)=e_{y, \text { equil. }}\left(1-e^{-\frac{t}{2 \tau_{y}}}\right)+\underbrace{e_{y, 0} e^{-\frac{t}{2 \tau_{y}}}}_{\begin{array}{c}
\text { memory of the initial } \\
\text { conditions is lost }
\end{array}}
$$


Table 2: Ring parameters, as set in the tracking simulations.

\begin{tabular}{|c|c|c|c|}
\hline 2 & Cell length & (m) & 50.800 \\
\hline 3 & Number of cells & & 16 \\
\hline 4 & Circumference, $\mathcal{C}=2 \pi R$ & $(\mathrm{~m})$ & 812.800 \\
\hline 5 & momentum compaction, $\alpha$ & $\left(10^{-4}\right)$ & 3.098 \\
\hline 6 & Qx & & 36.20 \\
\hline 7 & Qy & & 11.20 \\
\hline 9 & Q’x, Q’y, natural & & $-114,-34.5$ \\
\hline 10 & Q'x, Q'y, corrected & & $+0.035,-0.012$ \\
\hline \multicolumn{4}{|c|}{ Bend parameters : } \\
\hline 14 & $\mathrm{Nb}$. of bends & & 64 \\
\hline 15 & Bend deviation, $\theta$ & $(\mathrm{rad})$ & $2 \pi / 64$ \\
\hline 16 & Bend length, $\mathcal{L}$ & $(\mathrm{m})$ & 2.45 \\
\hline 17 & Curvature radius, $\rho$ & $(\mathrm{m})$ & 24.95549 \\
\hline \multicolumn{4}{|c|}{ Periodic functions at non-dispersive dipole end: } \\
\hline 18 & $\beta_{0}$ & $(\mathrm{~m})$ & 3.415 \\
\hline 19 & $\alpha_{0}$ & & 2.073 \\
\hline
\end{tabular}

Table 3: RF conditions, longitudinal parameters.

\begin{tabular}{lcc}
\hline Frequency, $f_{r f}=\omega_{r f} / 2 \pi$ & $(\mathrm{MHz})$ & 110.651 \\
Harmonic, $h$ & 300 \\
Synchronous phase, $\varphi_{s}$ & $(\mathrm{deg})$ & 30 \\
Peak voltage, $\hat{V}$ & $(\mathrm{MV})$ & $9.19123 \times\left(\frac{E_{s}[\mathrm{GeV}]}{6.000511}\right)^{4}$ \\
\hline
\end{tabular}

A remark on the accuracy of the simulation : the presence of transverse excitation (photon emission angle, assumed zero in the present ray-tracing simulations) would yield $\epsilon_{y} \rightarrow \frac{13}{55} \frac{C_{q}}{J_{y} I_{2}} \oint \frac{\beta_{y}}{\left|\rho^{3}\right|} d s \approx p m$, whereas, Fig. 3, the motion keeps damping in the present simulation, with constant damping time $\tau=\frac{T_{\text {rev }} E_{s}}{U_{s} J_{y}}$ (the emittance is quasi-zero here, $\epsilon_{y} / \pi \approx 1.510^{-41} \mathrm{~m}$, after 20000 turns, 15 damping times about, cf. Tab. 5).

\section{Concentration ellipses}

The comparisons discussed here abundantly lean on the concentration ellipses. In order to make things clear, we recall briefly the way these are computed.

Let $z_{i}(s), z_{i}^{\prime}(s)$, be the position and angle of the $i=1, n$ particles observed at some turn $(z=x, y$ or $\Delta E$ for respectively the horizontal, vertical or longitudinal motion).

The second order moments of the centered variables $z_{i}=z_{i}(s)-\bar{z}(s), z_{i}^{\prime}=z_{i}^{\prime}(s)-\overline{z^{\prime}}(s)$ are

$$
\begin{gathered}
\overline{z^{2}}(s)=\frac{1}{n} \sum_{i=1}^{n}\left(z_{i}(s)-\bar{z}(s)\right)^{2} \\
\overline{z z^{\prime}}(s)=\frac{1}{n} \sum_{i=1}^{n}\left(z_{i}(s)-\bar{z}(s)\right)\left(z_{i}^{\prime}(s)-\overline{z^{\prime}}(s)\right) \\
\overline{z^{\prime 2}}(s)=\frac{1}{n} \sum_{i=1}^{n}\left(z_{i}^{\prime}(s)-\overline{z^{\prime}}(s)\right)^{2}
\end{gathered}
$$

From these, a concentration ellipse (CE) is drawn (e.g., Fig. 3, right), with surface $\mathcal{S}_{z}(s)$ and equation

$$
\gamma_{c}(s) z^{2}+2 \alpha_{c}(s) z z^{\prime}+\beta_{c} z^{\prime 2}=\mathcal{S}_{z}(s) / \pi
$$



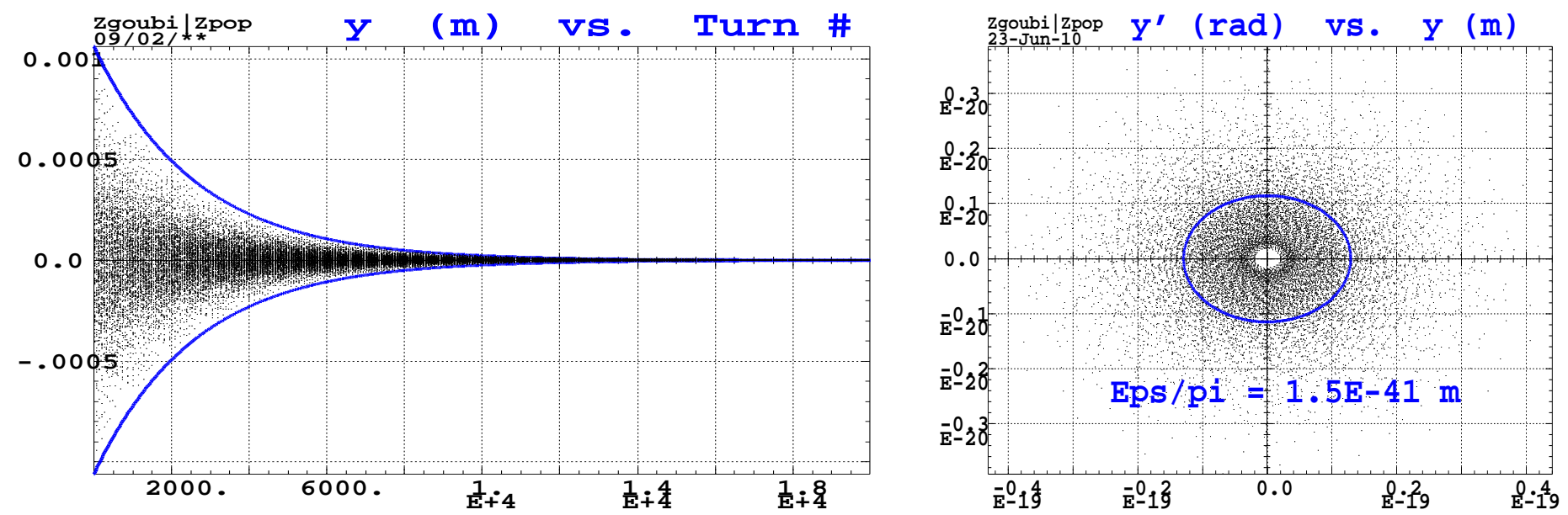

Figure 3: Damping of vertical motion over 20000 turns (left plot). A single particle is tracked. Its vertical invariant decreases toward zero value (transverse phase space in the right plot, last 1000 turns), since the stochastic photon emission angle is not accounted for, in the present tracking.

Noting $\Delta=\overline{z^{2}}(s) \overline{z^{\prime 2}}(s)-{\overline{z z^{\prime}}}^{2}(s)$, the ellipse parameters write

$$
\gamma_{c}(s)=\overline{z^{\prime 2}}(s) / \sqrt{\Delta}, \quad \alpha_{c}(s)=-\overline{z z^{\prime}}(s) / \sqrt{\Delta}, \quad \beta_{c}(s)=\overline{z^{2}}(s) / \sqrt{\Delta}
$$

With these conventions, the rms values of $z$ and $z^{\prime}$ projections satisfy

$$
\sigma_{z}=\sqrt{\beta_{z} \mathcal{S}_{z} / \pi} \text { and } \sigma_{z^{\prime}}=\sqrt{\gamma_{z} \mathcal{S}_{z} / \pi}
$$

Note that, the accuracy to which the expected property $\beta_{c} \gamma_{c}-\alpha_{c}^{2}=1$ is satisfied provides an estimate of the quality of the statistics.

In addition, in the first order formalism, in non-dispersive regions, the $\mathrm{CE}$ surface identifies with the emittance,

$$
\mathcal{S}_{z}(s) / \pi=\epsilon_{z} / \pi
$$

\section{Damping simulations}

A number of multi-turn tracking simulations, at the manner of Fig. 3, have been performed. They are summarized in the following, in the form of a few figures and a series of tables.

App. D displays the present Chasman-Green lattice parameters as delivered by Zgoubi stepwise ray-tracing. Excerpts of typical input and output Zgoubi data in these Monte Carlo simulations are given and briefly commented in App. E.

Tracking starts with a large emittance (just to clearly see the damping, a different choice could have been to start from zero emittance and let it grow). The tracking is carried out up to equilibrium emittance, i.e., a few damping times away, whereas the concentration ellipse surface (CE-S) is computed turn after turn,

Four different energies have been considered : 6,9,12 and $18 \mathrm{GeV}$.

For all three motions, transverse and longitudinal, the evolution of the CE-S is considered to satisfy

$$
\epsilon(t)=\epsilon_{0} e^{-t / \tau}+\epsilon_{f}\left(1-e^{-t / \tau}\right)
$$

Fitting the Monte Carlo data to that formula (see Figs. 4, 5) yields the equilibrium emittance $\epsilon_{f}$, and damping time, $\tau$.

\section{A series of summary tables}

Results for $6 \mathrm{GeV}$ electron energy are displayed in Tabs. 4-7. 3000 or 6000 particles have been tracked, over 20000 or 40000 turns, depending on the exercise.

Several runs, however in rather limited number, have been performed in each case, with various initial conditions, random generator seeds, this will not be detailed here. Due to the limited number of trials, error bars can be up to a few percent level, depending on the parameters of concern, which is anyway considered encouraging as to the correct behavior of Zgoubi as to SR and its effects in rings.

In addition to Zgoubi tracking and to theoretical formulas, the light source code BETA [12] developed at Saclay is used in this benchmarking, as a follow on of the above mentioned initial work in Ref. [2]. Determination of SR parameters in BETA is based on the computation of the radiation integrals, from the lattice parameters, thus BETA's data are expected to agree with the theoretical formulas of concern, in these various tables. 
Table 4: SR integrals. In the "Theoretical" column, values are derived from the formulas shown on the left, these include the reduced expressions for an iso-magnetic lattice. In the "Ray-tracing" column, values have been derived indirectly, following the recipes given in footnote and using the numerical values of damping times and equilibrium emittances reported in Tabs. 5, 6, as obtained from Zgoubi multi-turn tracking in the 16-cell ring.

\begin{tabular}{|c|c|c|c|c|}
\hline \multicolumn{2}{|c|}{ ( $\oint \equiv$ int. over the dipoles) } & \multirow{2}{*}{$\begin{array}{l}\text { Units } \\
\text { (m) }\end{array}$} & \multirow{2}{*}{$\begin{array}{c}\text { Theoretical } \\
0.2516\end{array}$} & \multirow{2}{*}{$\begin{array}{c}\text { Ray-tracing } \\
0.250^{(c)}\end{array}$} \\
\hline$I_{1}=\oint \frac{D_{x}}{\rho(s)} d s$ & $=\alpha \mathcal{C}$ & & & \\
\hline$I_{2}=\oint \frac{d s}{\rho(s)^{2}}$ & $=\frac{2 \pi}{\rho}$ & $\left(\mathrm{m}^{-1}\right)$ & 0.2518 & $0.253^{(d)}$ \\
\hline$I_{3}=\oint \frac{d s}{|\rho(s)|^{3}}$ & & $\left(10^{-2} \mathrm{~m}^{-2}\right)$ & 1.0089 & $1.017^{(e)}$ \\
\hline$I_{4}=\oint \frac{D_{x}}{\left[\left.\rho(s)\right|^{3}\right.}(1-2 n) d s$ & $=\frac{1}{|\rho|^{3}} \oint D_{x} d s(a)$ & $\left(10^{-4} \mathrm{~m}^{-1}\right)$ & 4.26 & $\approx 5^{(f)}$ \\
\hline$I_{5}=\oint \frac{\mathcal{H}}{|\rho(s)|^{3}} d s$ & $=\frac{2 \pi}{|\rho|^{2}} \overline{\mathcal{H}}^{(b)}$ & $\left(10^{-5} \mathrm{~m}^{-1}\right)$ & 3.2562 & $3.271(g)$ \\
\hline$\overline{\mathcal{H}}=\frac{1}{2 \pi \rho} \oint_{\text {bends }} \mathcal{H} d s$ & $\stackrel{(C G)}{=} \rho \theta^{3}\left(\frac{\gamma_{0} \mathcal{L}}{20}+\frac{\beta_{0}}{3 \mathcal{L}}-\frac{\alpha_{0}}{4}\right)$ & $(\mathrm{mm})$ & 3.2209 & $3.207^{(h)}$ \\
\hline \multicolumn{5}{|c|}{$\begin{array}{l}\text { (a) } n=-\rho / B \partial B / \partial x \text { field index, zero here. } \\
\text { (b) } \mathcal{H}=\gamma_{x} D_{x}^{2}+2 \alpha_{x} D_{x} D_{x}^{\prime}+\beta_{x} D_{x}^{\prime 2} .(C G): \text { case of Chasman-Green lattice. } \\
\text { (c) From momentum compaction. } \\
\text { (d) From vertical damping time, } \tau_{\epsilon_{y}} \text {, Tab. } 5: I_{2}=3 T_{r e v} /\left(2 r_{0} \gamma^{3} \tau_{y}\right) \\
\text { (e) From } I_{2}: I_{3}=I_{2}^{2} /(2 \pi)=0.2528^{2} /(2 \pi) . \\
\text { (f) From } I_{2} \text { and damping parameter } \mathcal{D} \text {, Tab. } 7 . \\
\text { Notes : } \\
\quad \text { (i) higher accuracy on } \mathcal{D} \text { would require more statistics, } \\
\text { (ii) using damping times instead (Tab. 5) yields even greater uncertainty, since } I_{4} \sim D_{x} / \rho^{2} \ll I_{2} \sim 1 / \rho \text {. } \\
\text { (g) From } I_{2} \text { and from } I_{5} / I_{2} \text {, equilibrium } \epsilon_{x} \text {, Tab. } 6 \text {. } \\
\text { (h) From equilibrium } \epsilon_{x} \text {, Tab. } 6 .\end{array}$} \\
\hline
\end{tabular}

Table 5: Emittance damping times. Agreement between Zgoubi tracking and theoretical expectations is well within a percent, for the three motions. The values in the "Zgoubi" column are the averages over a few trials.

\begin{tabular}{|c|c|c|c|c|c|}
\hline & Units & BETA & Zgoubi & The & \\
\hline \multirow{3}{*}{ horizontal, $\tau_{\epsilon_{x}}$} & & code & & value & formula \\
\hline & $\mathrm{ms}$ & 3.546 & $3.547^{(a)}$ & 3.547 & \multirow{2}{*}{$=\frac{T_{r e v} E_{s}}{U_{s} J_{x}}=\frac{3 T_{r e v}}{2 r_{0} \gamma^{3}\left(I_{2}-I_{4}\right)}$} \\
\hline & turns & 1308 & 1308 & 1308 & \\
\hline \multirow{2}{*}{ vertical, $\tau_{\epsilon_{y}}$} & $\mathrm{~ms}$ & 3.540 & $3.547^{(a)}$ & 3.541 & \multirow{2}{*}{$=\frac{T_{r e v} E_{s}}{U_{s} J_{y}}=\frac{3 T_{r e v}}{2 r_{0} \gamma^{3} I_{2}}$} \\
\hline & turns & 1306 & 1308 & 1306 & \\
\hline \multirow[t]{2}{*}{ longitudinal, $\tau_{\epsilon_{l}}$} & $\mathrm{~ms}$ & 1.769 & $1.757^{(a)}$ & 1.769 & \multirow{2}{*}{$=\frac{T_{r e v} E_{s}}{U_{s} J_{l}}=\frac{3 T_{r e v}}{2 r_{0} \gamma^{3}\left(2 I_{2}+I_{4}\right)}$} \\
\hline & turns & 652 & 648 & 652 & \\
\hline
\end{tabular}

(a) Obtained by matching time-dependent CE-Surface with $\epsilon(t)=\epsilon_{0} e^{-t / \tau}+\epsilon_{\text {equil. }}\left(1-e^{-t / \tau}\right)$, see Sec. 4 . 
LONGITUD INAL

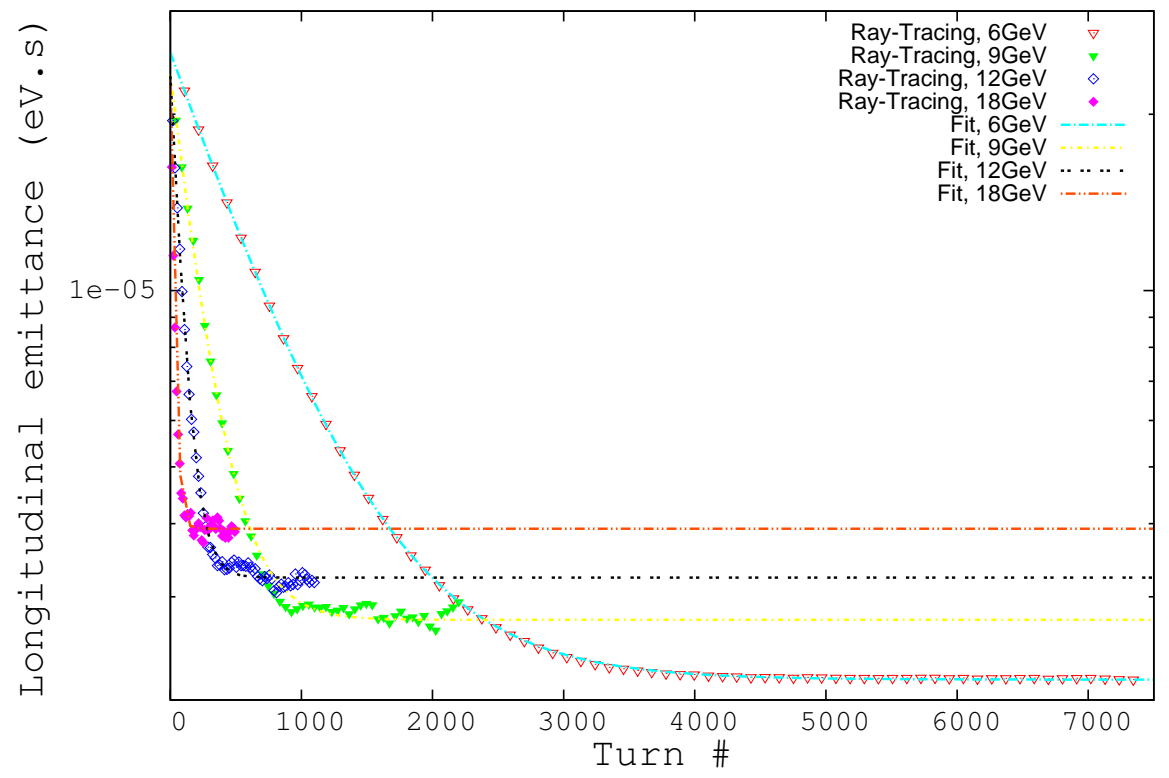

Figure 4: Evolution of the longitudinal emittance with the number of turns. Damping times in Tabs. 5, 8 have been determined from similar data, by matching with the exponential decay (Eq. 9).

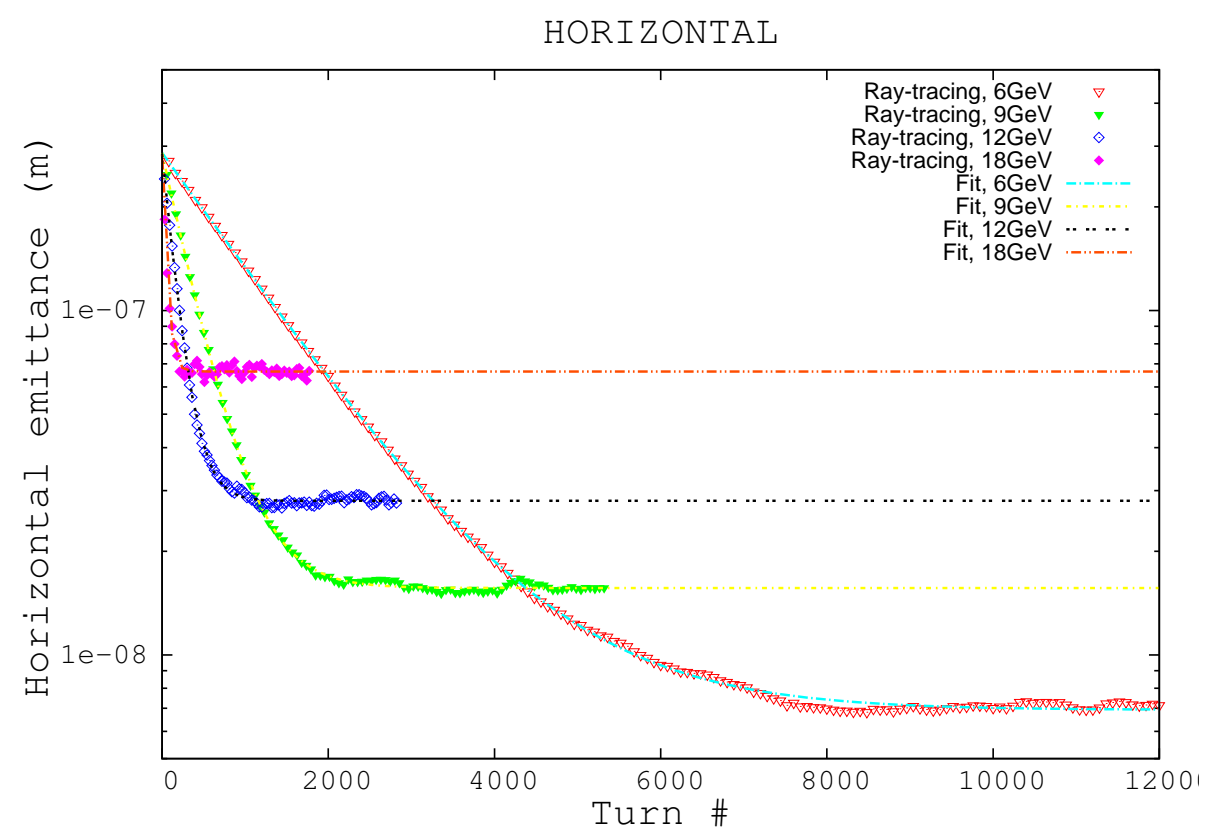

Figure 5: Evolution of the horizontal emittance with the number of turns. Damping times in Tab. 5, 8 have been determined from similar data, by matching with the exponential decay (Eq. 9). 
Table 6: Natural emittances and beam sizes. They are obtained by matching the turn-by-turn CE-S as in Figs. 4, 5, or coordinate excursions as in Fig. 3, with respectively Eq. 9 or Eq. 8.

\begin{tabular}{|c|c|c|c|c|c|}
\hline & Units & BETA & Zgoubi & \multicolumn{2}{|c|}{ Theoretical } \\
\hline & & code & tracking $(a)$ & value & formula \\
\hline horizontal, $\epsilon_{x}$ & $\mathrm{~nm}$ & 6.843 & 6.83 & 6.831 & $=\frac{\mathcal{C}_{q} \gamma^{2}}{J_{x}} \frac{I_{5}}{I_{2}} \stackrel{i s o-\rho}{=} \frac{\mathcal{C}_{q} \gamma^{2}}{J_{x} \rho} \overline{\mathcal{H}}(c)(d)$ \\
\hline vertical, $\epsilon_{y}$ & $\mathrm{pm}$ & - & $\ll p m{ }^{(b)}$ & $\approx 0.15$ & $=\frac{13}{55} \frac{C_{q}}{J_{y} I_{2}} \oint \frac{\beta_{y}}{\left|\rho^{3}\right|} d s$ \\
\hline longitudinal, $\epsilon_{l}=\sigma_{\frac{d \hat{E}}{E}}^{2}$ & $\mu \mathrm{eV} . \mathrm{s}$ & - & 2.22 & 2.117 & $=2 \frac{\mathcal{C}_{q} \gamma^{2}}{J_{l} \rho}$ \\
\hline $\operatorname{rms} d E / E, \sigma_{\frac{d E}{E}}=\frac{1}{\sqrt{2}} \sigma_{\frac{d \hat{d E}}{E}}$ & $10^{-3}$ & 1.03 & 1.023 & 1.058 & $=\sqrt{\frac{\mathcal{C}_{q}}{J_{l} \rho}} \gamma$ \\
\hline rms bunch length, $\sigma_{s}$ & $\mathrm{~mm}$ & - & 9.40 & 9.301 & $=\frac{\alpha c}{\Omega_{s}} \sigma_{\frac{d E}{E}}$ \\
\hline
\end{tabular}

(a) Statistical error bars on Zgoubi quantities are omitted, generally within percent.

(b) Transverse excitation is off in Zgoubi for these benchmarking simulations.

(c) $\mathcal{C}_{q}=\frac{55}{32 \sqrt{3}} \frac{\bar{h}}{m_{0} c} \approx 3.83193810^{-13}[\mathrm{~m}]$.

(d) This yields $J_{x}$ (Tab. 7), namely, $J_{x}=\frac{3.83193810^{-13} \times 11742.683^{2}}{\epsilon_{x} \times 24.9555[\mathrm{~m}]} \times 3.2209[\mathrm{~m}]=0.9984$

Table 7: Partition numbers, damping parameter. Average values from a few trials are given. In general the theoretical value falls within that set of values. More statistics would be required for better precision, e.g., so to satisfy $J_{y}=1$ with higher accuracy.

\begin{tabular}{|c|c|c|c|c|c|}
\hline & Units & BETA & Zgoubi & The & ical \\
\hline & & code & & value & formula \\
\hline$J_{x}$ & & 0.9984 & $0.998^{(a)}$ & 0.9984 & $=1-\mathcal{D} \stackrel{\text { strongfoc. }}{\longrightarrow} 1^{-}$ \\
\hline$J_{y}$ & & 1 & $0.998^{(b)}$ & 1 & $=1$ \\
\hline$J_{l}$ & & 2.0016 & $2.002^{(c)}$ & 2.0016 & $=2+\mathcal{D} \stackrel{\text { strongfoc. }}{\longrightarrow} 2^{+}$ \\
\hline \multirow[t]{2}{*}{ Damping parameter, $\mathcal{D}$} & $10^{-3}$ & 1.6049 & $1.95^{(d)}$ & 1.6049 & \multirow[t]{2}{*}{$=\frac{I_{4}}{I_{2}} \stackrel{\substack{n=0 \\
i s o-\rho}}{=} \frac{\alpha \mathcal{C}}{2 \pi \rho}$} \\
\hline & $10^{-3}$ & 1.6049 & $2^{(d)}$ & 1.6049 & \\
\hline
\end{tabular}

(a) From $\tau_{\epsilon_{x}}$ value, Tab. $5: J_{x}=\frac{T_{r e v} E_{s}}{U_{s} \tau_{\epsilon_{x}}}=\frac{2.7112310^{-6} \times 6000.511}{4.5956 \times \tau_{x}}=\frac{3.54010^{-3}}{3.54710^{-3}}$.

(b) From $\tau_{\epsilon_{y}}$ value : $J_{y}=\frac{3.54010^{-3}}{3.54710^{-3}}$, rms value at few $10^{-3}$ level. Deserves more statistics.

(c) From $\sigma_{\frac{d E}{E}}$ or $\tau_{\epsilon_{l}}$ values.

(d) Values obtained from, respectively, $\mathcal{D}=1-J_{x}, J_{l}-2$. 


\section{Energy dependence}

Benchmarking for energy dependence of equilibrium emittances and damping times is summarized in Tab. 8. Expected $\gamma$-scaling laws are recalled (3rd row), as well as energy loss (2nd column). Values between square brackets are the theoretical, expected, ones. A limited number of tracking trials have been realized in general for any of these quantities, their average value is displayed in the table. In spite of that, the agreement is rather satisfactory, within a few percent or better. Differences with formulæ may have various origins : horizontal oscillation due to synchrotron motion upon mis-centering of the beam at injection, global fluctuations of beam size with turn number due to the limited stastitics (a few hundred to a few thousand particles), etc.

Table 8: Energy dependence of energy loss, and of longitudinal and horizontal emittances and damping times. It can be checked that the theoretical $\gamma$-scaling rules are satisfied.

\begin{tabular}{lccccc}
\hline & $\begin{array}{c}\text { Energy loss, } U_{s} \\
(\mathrm{MeV})\end{array}$ & $\begin{array}{c}\epsilon_{l} \\
(\mu \mathrm{eV} . \mathrm{s})\end{array}$ & $\begin{array}{c}\tau_{l} \\
(\mathrm{~ms})\end{array}$ & $\begin{array}{c}\epsilon_{x} \\
(\mathrm{~nm})\end{array}$ & $\begin{array}{c}\tau_{x} \\
(\mathrm{~ms})\end{array}$ \\
Scaling law & $\gamma^{\mathbf{4}}$ & $\gamma^{\mathbf{1 / 2}}$ & $\mathbf{1} / \gamma^{\mathbf{3}}$ & $\gamma^{\mathbf{2}}$ & $\mathbf{1} / \gamma^{\mathbf{3}}$ \\
$6 \mathrm{GeV}$ & $4.5956[4.5941]$ & $2.22[2.12]$ & $1.76[1.7690]$ & $6.83[6.83]$ & $3.55[3.5466]$ \\
$9 \mathrm{GeV}$ & $23.257[23.257]$ & $2.74[2.717]$ & $0.575[0.5242]$ & $15.6[15.37]$ & $1.02[1.0508]$ \\
$12 \mathrm{GeV}$ & $73.505[73.505]$ & $3.24[3.137]$ & $0.222[0.2211]$ & $28.0[27.32]$ & $0.447[0.4433]$ \\
$18 \mathrm{GeV}$ & $372.12[372.21]$ & $3.92[3.842]$ & $0.0676[0.0655]$ & $66.5[61.46]$ & $0.135[0.1314]$ \\
& & & & & \\
\hline
\end{tabular}




\section{Coupled motion}

\subsection{Working hypotheses}

The stochastic change of direction of momentum vector is "off" in Zgoubi in the present simulations. The source of vertical emittance is a single skew quadrupole introduced in a dispersion free drift.

The difference resonance $Q_{x}-Q_{y}-i n t .=\Delta=0$ is considered. The coupling coefficient is

$$
\kappa=\frac{1}{2 \pi} \oint K_{s} \sqrt{\beta_{x} \beta_{y}} e^{i\left(\psi_{x}-\psi_{y}-\left(Q_{x}-Q_{y}+i n t .\right) 2 \pi s / \mathcal{C}\right)} d s
$$

with $K_{s}$ the skew quadrupole strength.

As a consequence, in the weak coupling approximation, and accounting for the present definition of the 2-D CE-Surface (section 4),

- the ratio of the equilibrium beam emittances is expected to satisfy

$$
\frac{\epsilon_{y}}{\epsilon_{x}}=\frac{\kappa^{2}}{\kappa^{2}+\Delta^{2}}
$$

- the sum of the invariants is expected to remain constant, equal to the natural emittance,

$$
\epsilon_{x}+\epsilon_{y}=\epsilon_{0}, \quad \epsilon_{0}=\text { uncoupled natural emittance }
$$

\subsection{Typical data out of Zgoubi}

Series of 3000 or 6000 particles are tracked for 20000 turns. From their coordinates, CE-S are derived, as a function of turn number. The tracking is iterated for various values of the coupling strength. The graph below is a typical result so obtained, it shows the evolution of the horizontal and vertical CE-S for a particular coupling strength.

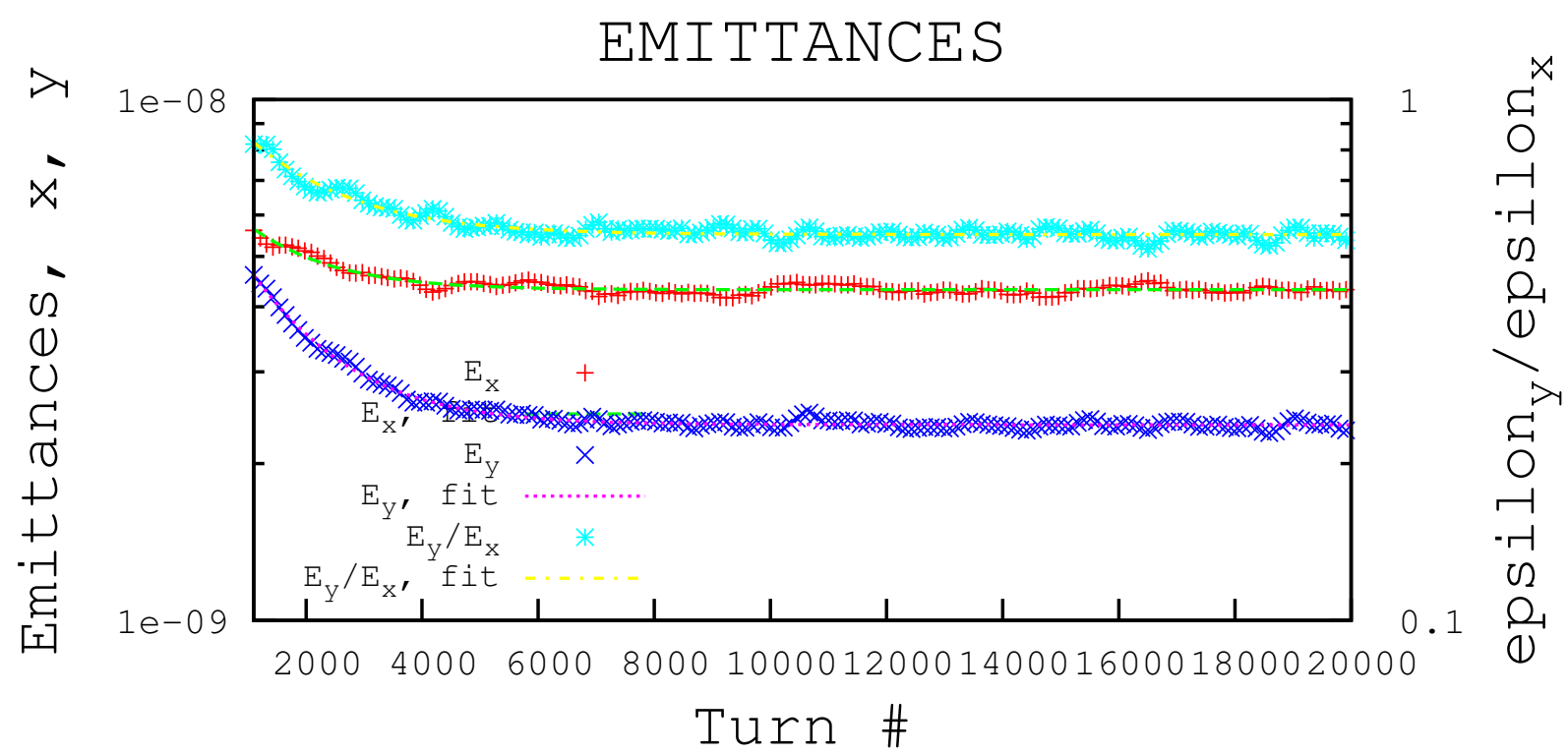

Figure 6: Horizontal and vertical emittances in presence of coupling, their ratio, as a function of the number of turns, from Zgoubi tracking, and a fit by the exponential decay law, This is similar to Fig. 5, it differs by the presence of coupling.

For the evolution of both $\epsilon_{x}$ and $\epsilon_{y}$ with time, the interpolation function is of the form

$$
\epsilon(t)=\epsilon_{0} e^{-t / \tau}+\epsilon_{f}\left(1-e^{-t / \tau}\right)
$$

\subsection{Outcome of the numerical tracking}

Numerical simulations yield the discrete data (markers) shown in Fig. 6, and their interpolation by the theoretical law

$$
\frac{\epsilon_{y}}{\epsilon_{x}}=\frac{\kappa^{2}}{\kappa^{2}+\Delta^{2}}
$$

In the weak coupling region of the graph, it can be observed that the concentration ellipse surfaces satisfy $\left(\epsilon_{x}+\epsilon_{y}\right) / \epsilon_{0} \approx 1$, as expected [13]. 


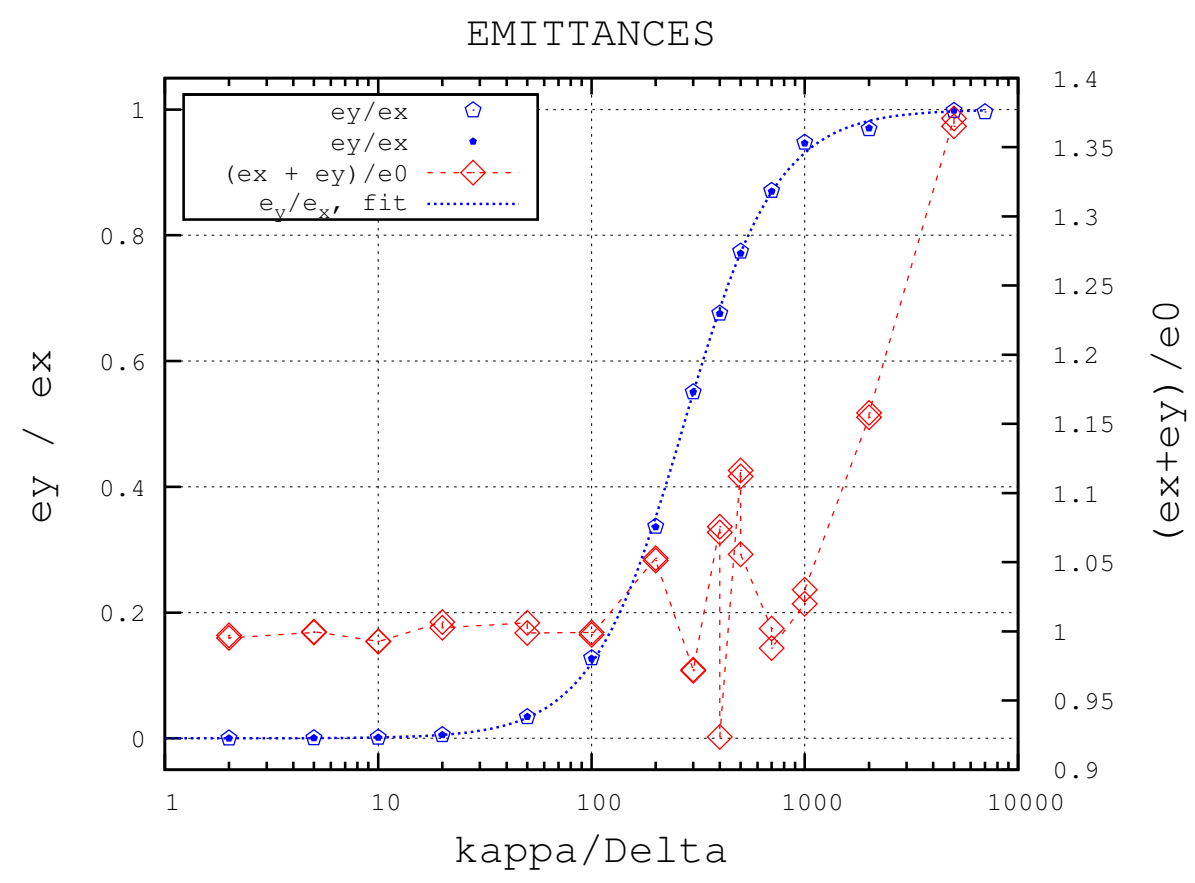

Figure 7: Left vertical scale : ratio of vertical to horizontal emittances, from Zgoubi tracking (markers), and their interpolation by the theoretical law $\frac{\epsilon_{y}}{\epsilon_{x}}=\frac{\kappa^{2}}{\kappa^{2}+\Delta^{2}}$ ("fit" curve). Right scale : $\left(\epsilon_{x}+\epsilon_{y}\right) / \epsilon_{0}$ from these Zgoubi tracking : that ratio comes out constant in the weak coupling limit, as expected, it becomes erratic, and anyway lacks statistics, beyond $\kappa / \Delta>100$.

\section{CPU time considerations}

The simulations discussed were realized on a $2.5 \mathrm{GHz}$ processor. The real time consumption, in cruise regime, large number of particles and large number of turns, with $1 \mathrm{~cm}$ integration step in all magnets (far too small, however a choice aimed at ensuring convergence of Zgoubi numerical integration), is

$20 \mathrm{~ms} /$ particle / turn, in that 800 long structure.

This can be roughly extrapolated to the eRHIC case, as follows :

(i) a $3800 \mathrm{~m}$ long structure, a factor 5 about

(ii) assuming 10 passes in the e-ring, an additonal factor 10

which means 1 second about per particle, i.e., 24 hours about for $10^{5}$ particles for good statistics. Or as well series of 1-2 hours per run on a desk computer for lattice parameter optimization trials, for instance.

Adding spin tracking won't change the CPU time much, an additional $20 \%$ about. Note that a $1 \mathrm{~cm}$ integration step should be small enough to warrant the accuracy (and sufficient symplecticity) on spin tracking, given that $G \gamma$, the number of spin precessions in a turn in RHIC, is 22 about $\left(10 \mathrm{GeV}\right.$, electron $G \approx 1.1 \times 10^{-3}$ ), i.e., less than a full precession, in a single bending magnet, at highest energy (for reference, $1 \mathrm{~cm}$ is the step size used for tracking - successfully - $250 \mathrm{GeV}$ polarized protons in RHIC [14], where they reach $G \gamma \approx 455$ at top energy).

\section{Comments / conclusions}

High accuracy stepwise ray-tracing of 6-D motion in Zgoubi in presence of SR seems established. The computational material for further spin diffusion benchmarking against theoretical expectations is there, a follow on is essentially a matter of additional simulations.

The benchmarking conditions here however, thousands of turns in rings, are challenging beyond necessity regarding simulations planned in eRHIC, namely a few passes in a $3.8 \mathrm{~km}$ long recirculating type of accelerator structure.

Worth inspecting further on the other hand, or keeping an eye on :

- effects of scattering, which are not addressed here. In particular, and also a test of accuracy : decay of $\epsilon_{y}$ towards minimal invariant in presence of transverse scattering,

- conservation of the average values of Eigen invariants, $\epsilon_{I-I V}$, in presence of strong coupling.

- beam dynamics in presence of synchrotron radiation in all magnets. 


\section{APPENDIX}

\section{A SR in Zgoubi, earlier benchmarking}

\section{A.1 Tesla Test Facility BDS}

Some essential aspects of Zgoubi outcomes are recalled regarding SR effects in beam lines, namely here the Tesla Test Facility beam delivery system. The details of the code developments and their application to lepton beam lines can be found in Ref. [2].

As an illustration, the two plots below show the growth of the concentration ellipse surface (the quantity of concern is recalled in detail in Sec. 4, p. 7), at IP, due to SR in the TTF BDS. $210^{4}$ particles where tracked. The numbering of the curves in the left plot correspond to, (1) zero initial emittances, (2) nominal 6-D emittance, (3) chromaticity corrected optics.

The next six plots illustrate two types of deleterous effects, as follows.

A fosused beam at the IP is considered, left column, assuming (i) SR in the sole bends and (ii) scaled magnet fields so to follow theoretical average beam rigidity decrease along the BDS. Then, defocusing is observed (middle column) if the magnetic field in the quadrupoles is, instead, not scaled to the energy loss along the line. Defocusing is also observed (right column) if SR is accounted for in all magnets (quadrupoles and sextupoles, in addition to the dipoles).

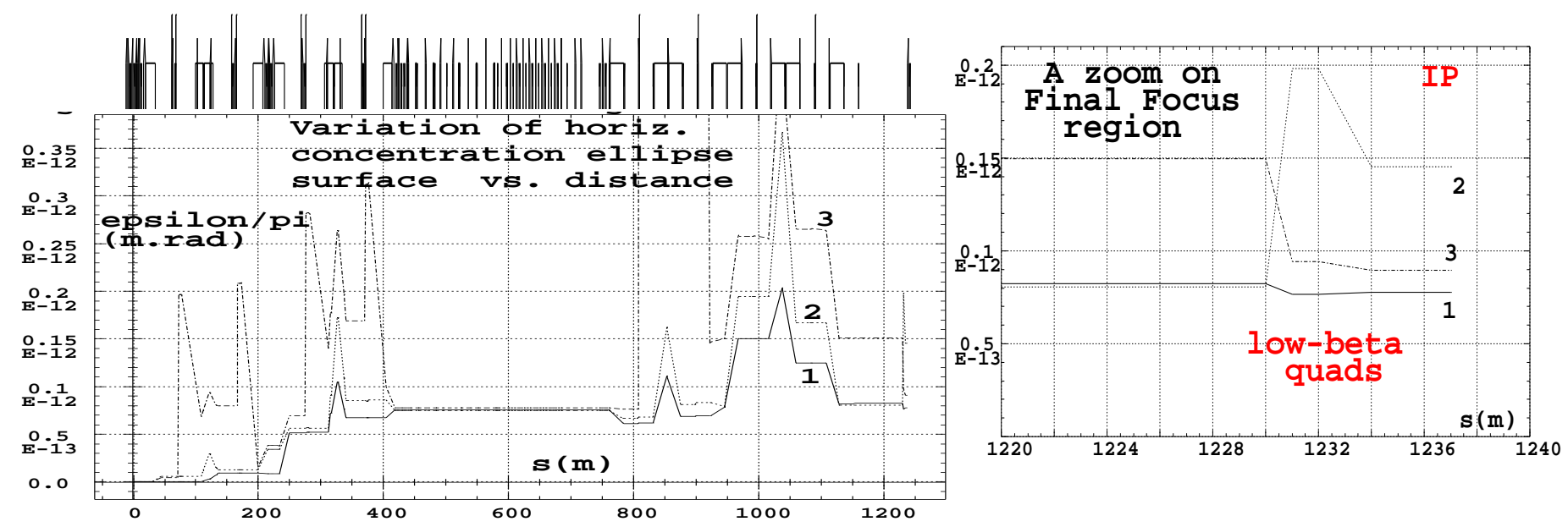

Field scaling set, SR in bends only.

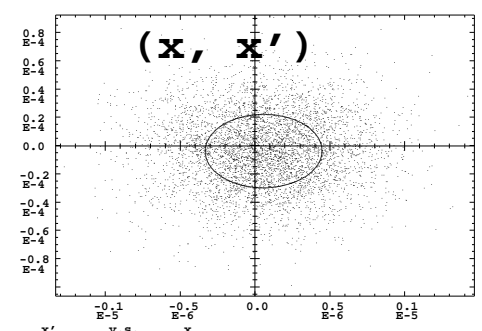

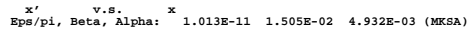

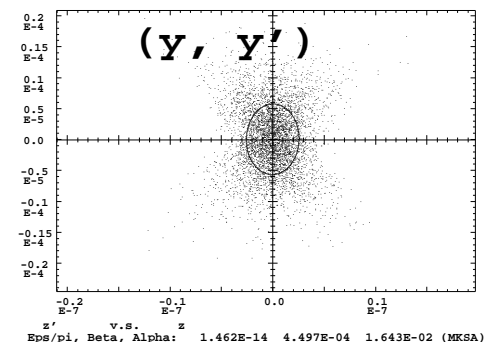

Mis-focusing at FF, due to quadrupoles not scaled

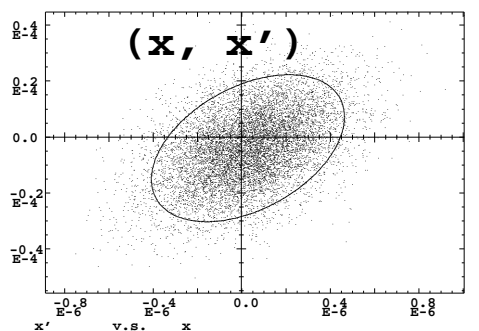

$\mathbf{x}^{\prime}$
Eps/pi, Beta,
V.s.

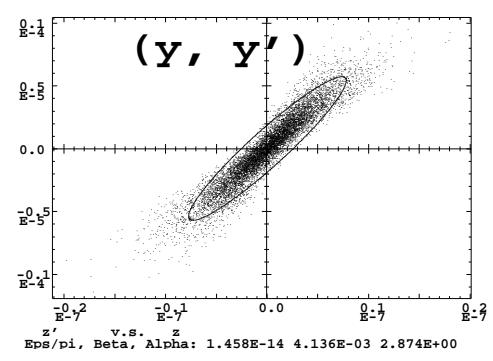

Field scaling set in all magnets, SR set in all magnets.

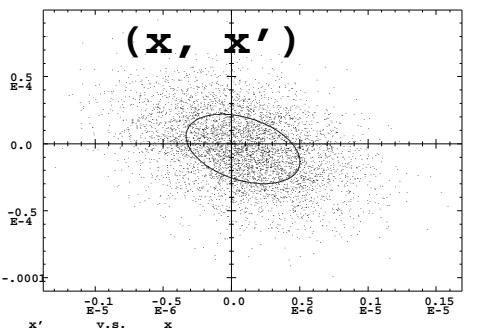

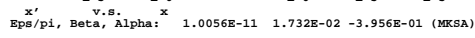

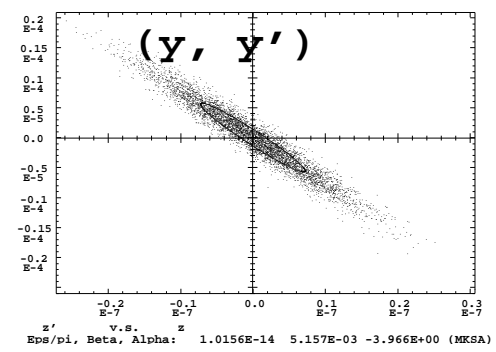




\section{A.2 Super-B rings}

An early application of these newly developed SR damping simulations in rings concerned the super-B project, an assymmetric $4 \times 7 \mathrm{GeV}$ collider, with the goal of, later, including spin transport [9]. The figures below show the optical settings in the high energy ring (HER), as of Zgoubi.
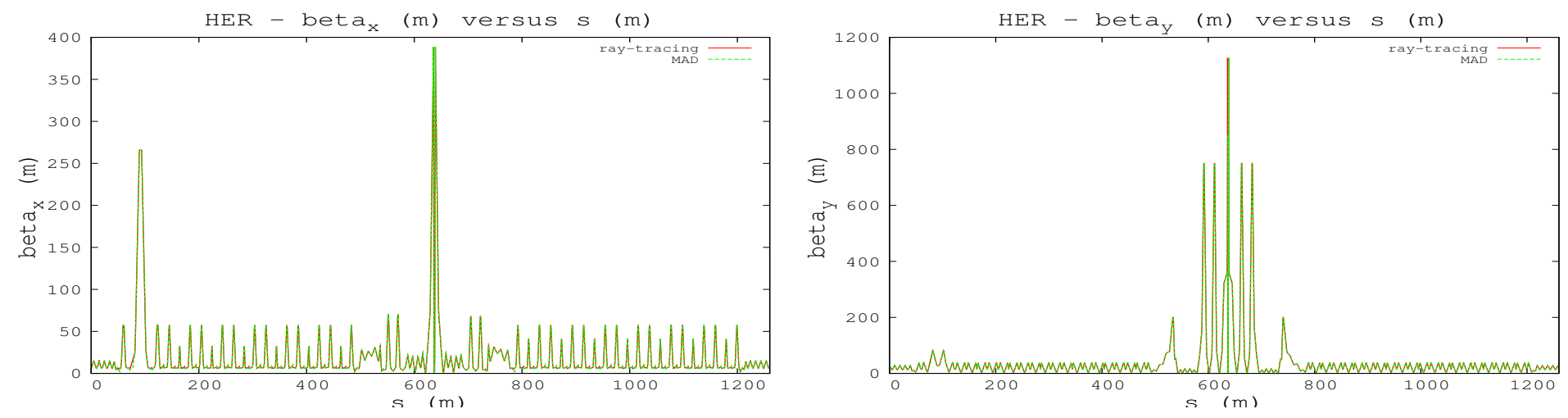

The left table below summarizes the LER and HER ring parameters, the top right one gives the main, theoretical, SR related quantities.

The lower right table displays typical beam dynamics parameters resulting from SR. The values for the natural emittance $\left(\epsilon_{x} / \pi\right)$ and longitudinal damping time ( $\tau_{E}$ ) from Zgoubi tracking (rightmost column) differ from the published expected quantities (left column). Regardless of the detailed reasons for these disagreements (they were unfortunately not investigated in detail at that time, they may have to be looked for in the iso-magnetic hypothesis in the theoretical data, or in the SR in all magnets on Zgoubi side), that was an early motivation for undertaking tight benchmarking investigations.

- Super-B rings parameters, comparing MAD8 and Zgoubi data.

\section{HER}

Energy /GeV

Orbit length $/ \mathrm{m}$

$Q_{x}, Q_{y}$

$Q_{x}^{\prime}, Q_{y}^{\prime}$

$\alpha, \sqrt{1 / \alpha}$

$\operatorname{Max} \beta_{x}, \beta_{y} / \mathrm{m}$

Max Dx /m

\section{LER}

Energy /GeV

Orbit length $/ \mathrm{m}$

$Q_{x}, Q_{y}$

$Q_{x}^{\prime}, Q_{y}^{\prime}$

$\alpha, \sqrt{1 / \alpha}$

$\operatorname{Max} \beta_{x}, \beta_{y} / \mathrm{m}$

$\operatorname{Max} \mathrm{Dx} / \mathrm{m}$
MAD8

\section{Zgoubi}

6.7

1258.3581

$40.5750,17.5950$

$0.042,-0.0038$

$4.36110^{-4}, 47.88$

$388.57,1126.40$

0.6346

1258.3582

$40.5750,17.5950$

$0.062,-0.0019$

$4.37110^{-4}, 47.83$

$388.55,1126.35$

0.6346

4.18

1258.3581

42.5744, 18.6019

$-0.620,-0.678$

$4.04910^{-4}, 49.69$

$396.54,1013.17$

0.5118
1258.3582

$42.5749,18.5949$

$-0.624,-0.676$

$4.05310^{-4}, 49.67$

$387.25,1146.77$

0.5118

- Theoretical SR parameters in LER, based on radiation in bends only and iso-magnetic lattice.

\begin{tabular}{lcc}
\hline Synchronous energy $E_{s}$ & $\mathrm{GeV}$ & 4.18 \\
Orbit length & $\mathrm{m}$ & 1258 \\
Orbit duration & $\mu \mathrm{s}$ & 4.20 \\
Average energy loss /particle/turn, $E_{\text {loss }}$ & $\mathrm{keV}$ & 864.84 \\
Equivalent $\rho: 88.463 \frac{E^{4}[\mathrm{GeV}]}{E_{\text {loss }}[\mathrm{keV}]}$ & $\mathrm{m}$ & 31.2 \\
$\hat{V} \sin \left(\phi_{s}\right)$ & $\mathrm{kV}$ & 865 \\
\hline
\end{tabular}

- SR in LER. Col. 3 : theoretical, in bends only, assuming iso-magnetic lattice. Col. 4 : Zgoubi tracking, SR in all magnets.

\begin{tabular}{|lccc|}
\hline & & Theoretical & Zgoubi \\
$E_{\text {loss }}$ & $\mathrm{keV} /$ turn & 865.1 & 878.4 \\
$N_{\text {phot }}$ & & 541.4 & 541.3 \\
$\epsilon_{x} / \pi$ & $\mathrm{m}$ & $1.810^{-9}$ & $1.410^{-9}$ \\
$\tau_{x}$ & $\mathrm{~ms}$ & & $\approx 28$ \\
$\sigma_{E} / E$ & & $0.6710^{-3}$ & $0.6810^{-3}$ \\
$\tau_{E}$ & $\mathrm{~ms}$ & 20.3 & $\approx 15$ \\
\hline
\end{tabular}




\section{B Zgoubi method}

Details can be found in Zgoubi users' guide, Ref. [1].

Motion Zgoubi pushes particles step by step, with steps in $\sim \mathrm{cm}$ to $\sim 10 \mathrm{~s} \mathrm{~cm}$ range, depending on the problem.

Thus the equation which Zgoubi integrator solves is

$$
d(m \vec{v})=q(\vec{e}+\vec{v} \times \vec{b}) d t
$$

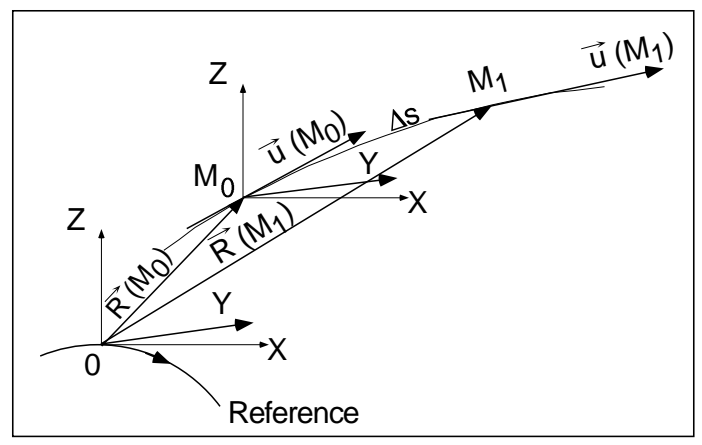

It is solved using truncated Taylor series in $\vec{R}$ and $\vec{u}=\vec{v} / v\left(\left(^{*}\right)^{\prime}=\mathrm{d}(*) / \mathrm{ds}\right)$ :

$$
\begin{aligned}
& \vec{R}\left(M_{1}\right) \approx \vec{R}\left(M_{0}\right)+\vec{u}\left(M_{0}\right) \Delta s+\vec{u}^{\prime}\left(M_{0}\right) \frac{\Delta s^{2}}{2 !}+\ldots+\vec{u}^{\prime \prime \prime \prime \prime}\left(M_{0}\right) \frac{\Delta s^{6}}{6 !} \\
& \vec{u}\left(M_{1}\right) \approx \vec{u}\left(M_{0}\right)+\vec{u}\left(M_{0}\right) \Delta s+\vec{u}^{\prime \prime}\left(M_{0}\right) \frac{\Delta s^{2}}{2 !}+\ldots+\vec{u}^{\prime \prime \prime \prime \prime}\left(M_{0}\right) \frac{\Delta s^{5}}{5 !}
\end{aligned}
$$

Fields The derivatives of the velocity, $d^{n} \vec{u} / d s^{n}$, needed in the Taylor series above, are computed from the magnetic field and its derivatives, $d^{n-1} \vec{B} / d s^{n-1}$ (noting $\vec{B}=\vec{b} / B \rho$, and assuming, here, $\vec{e}=0$ )

$$
\begin{aligned}
& \vec{u}^{\prime \prime}=\vec{u}^{\prime} \times \vec{B}+\vec{u} \times \vec{B}^{\prime} \\
& \vec{u}^{\prime \prime \prime}=\vec{u}^{\prime \prime} \times \vec{B}+2 \vec{u}^{\prime} \times \vec{B}^{\prime}+\vec{u} \times \vec{B}^{\prime \prime} \\
& \vec{u}^{\prime \prime \prime \prime}=\vec{u}^{\prime \prime \prime} \times \vec{B}+3 \vec{u}^{\prime \prime} \times \vec{B}^{\prime}+3 \vec{u}^{\prime} \times \vec{B}^{\prime \prime}+\vec{u} \times \vec{B}^{\prime \prime \prime} \\
& \text { etc. }
\end{aligned}
$$

Fields and derivatives are provided using either analytical models of fields, or 1- to 3-D field maps.

\section{Simulation of energy loss}

Probability of emission of a photon Given that the number of photons radiated within an integration step $\Delta s$ is normally small (units or fraction of a unit) ${ }^{5}$ a Poisson law

$$
p(k)=\frac{\Lambda^{-k}}{k !} e^{-k} \quad \text { with } \quad \Lambda=<k>, \Lambda=<(\Delta k)^{2}>
$$

is accounted for. $k$ is the number of photons radiated over an arc of trajectory $\Delta \theta$ such that, the average number of photons over an integration step expresses as ${ }^{6}$

$$
<k>=\Lambda=\frac{20 e r_{0}}{8 \hbar \sqrt{3}} \beta^{2} B \rho \Delta s
$$

with $r_{0}=e^{2} / 4 \pi \epsilon_{0} m_{0} c^{2}$ the classical radius of the particle of rest-mass $m_{0}, e$ the elementary charge, $\hbar=h / 2 \pi, h$ the Planck constant, $\beta=v / c, B \rho$ the particle stiffness and $\Delta s=\rho \Delta \theta$. At each integration step, $\Lambda$ is first evaluated from the current values of $\beta, B \rho$ and $\Delta s$, then a value of $k$ is drawn from Eq. 12 using a rejection method.

Energy of the photons These k photons are assigned energies $\epsilon=h \nu$ at random, in the following way. The cumulative distribution of the energy probability law $p\left(\epsilon / \epsilon_{c}\right) d \epsilon / \epsilon_{c}$, i.e., the probability that the photon has its energy in $\left.] 0, \epsilon\right]$, writes (Fig. below)

$$
\mathcal{P}\left(\epsilon / \epsilon_{c}\right)=\frac{3}{5 \pi} \int_{0}^{\epsilon / \epsilon_{c}} \int_{\epsilon / \epsilon_{c}}^{\infty} K_{5 / 3}(x) d x
$$

with $K_{5 / 3}$ the modified Bessel function, $\epsilon_{c}=\hbar \omega_{c}, \omega_{c}=3 \gamma^{3} c / 2 \rho$ the critical frequency of the radiation. The latter is evaluated at each integration step ${ }^{7}$ from the current values of $\gamma$ and $\rho$. In the low frequency region $\left(\epsilon / \epsilon_{c}<10^{-2}\right), \mathcal{P}\left(\epsilon / \epsilon_{c}\right.$ (Eq. 14) is approximated in Zgoubi (at better than $1 \%$ precision) using

$$
\mathcal{P}\left(\epsilon / \epsilon_{c}\right)=\frac{12 \sqrt{3}}{2^{1 / 3} 5 \Gamma\left(\frac{1}{3}\right)}\left(\epsilon / \epsilon_{c}\right)^{1 / 3}
$$

Beyond, over $10^{-2}<\epsilon / \epsilon_{c} \leq 10, \mathcal{P}\left(\epsilon / \epsilon_{c}\right)$ is interpolated from a set of 40 values that have been tabulated in Zgoubi source software.

\footnotetext{
${ }^{5}$ For instance, a $10 \mathrm{GeV}$ electron will radiate on average $\Lambda=20.038 E[\mathrm{GeV}] \times \Delta \theta \approx 206 \times \Delta \theta$ photons over a step $\Delta s=\rho \Delta \theta$, i.e., about 2 photons for a $10 \mathrm{~cm}$ step under the effect of a $10 \mathrm{~m}$ radius bending.

${ }^{6}$ This leads for instance to the usual formula for electrons : $\Lambda / \Delta \theta[\mathrm{rad}] \approx 129.5 \mathrm{E}[\mathrm{GeV}] / 2 \pi \approx \gamma / 94.9$.

${ }^{7}$ From a practical viewpoint, the value of the magnetic field is first computed for a one-step push of the particle, and then used to obtain $\rho$, following what, SR loss corrections are applied.
} 
Thus, In order to get $\epsilon / \epsilon_{c}$, a random value $0<\mathcal{P}<1$ is first generated uniformly, then $\epsilon / \epsilon_{c}$ is drawn from Eq. 15 if $\mathcal{P}<0.26$, or by inverse linear interpolation from the tabulated values if $\mathcal{P}>0.26$.

Note that - a checking means, see Tab. 1 - in a uniform magnetic field statistics is expected to converge towards the following ensemble averages :

- critical energy $\epsilon_{c}(e V)=3 \hbar \gamma^{3} c / 2 \rho e$,

- average photon energy $\bar{\epsilon}=8 \epsilon_{c} / 15 \sqrt{3}$,

- rms energy width $\left(\overline{\epsilon^{2}}-\bar{\epsilon}^{2}\right)^{1 / 2}=\sqrt{211 / 675} \epsilon_{c}$,

- number of average photons per particle $=\Delta E / \bar{\epsilon}$.

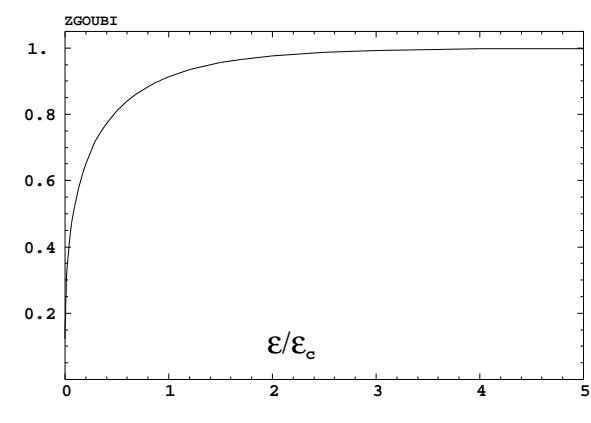

Cumulative distribution $\mathcal{P}\left(\epsilon / \epsilon_{c}\right)$.

\section{Chasman-Green lattice parameters, out of Zgoubi}

These details aim at making the working conditions clear, regarding the present benchmarking simulations.

All the figures are produced from the stepwise ray-tracing. Zgoubi has a dedicated toolbox for that. Note that, as expected, in the paraxial approximation (small angles) Zgoubi delivers the same data as would be obtained using any matrix code.

\section{Optical functions}
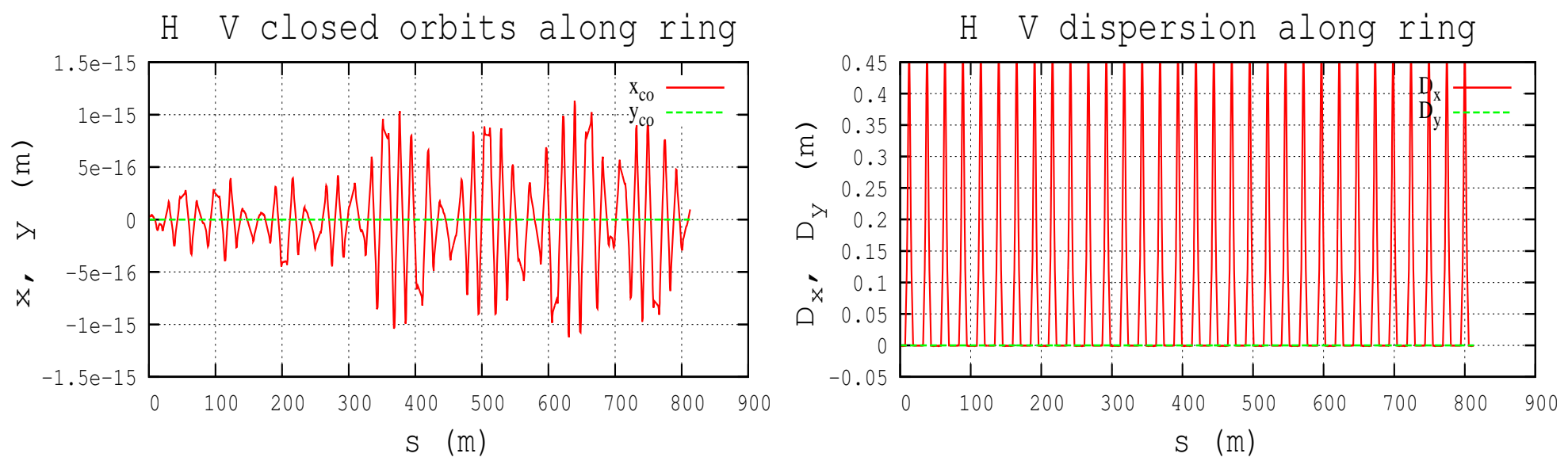

Figure 8: Left : closed orbits, $\mathrm{H}$ and V. Right : dispersion.
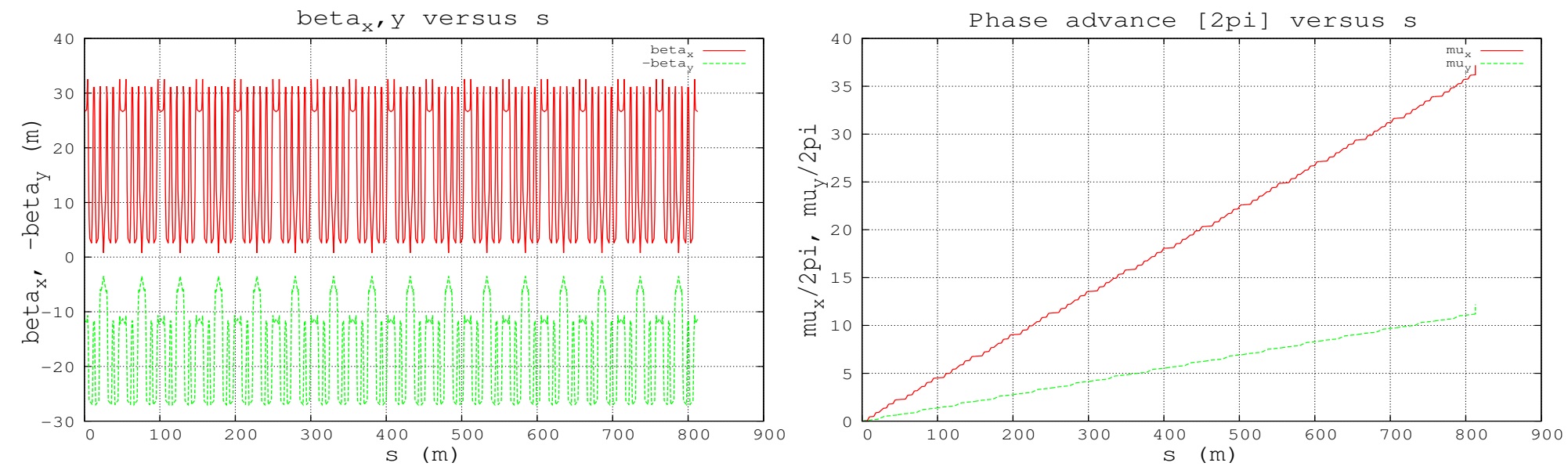

Figure 9: Left : betatron functions. Right : phase advance. 

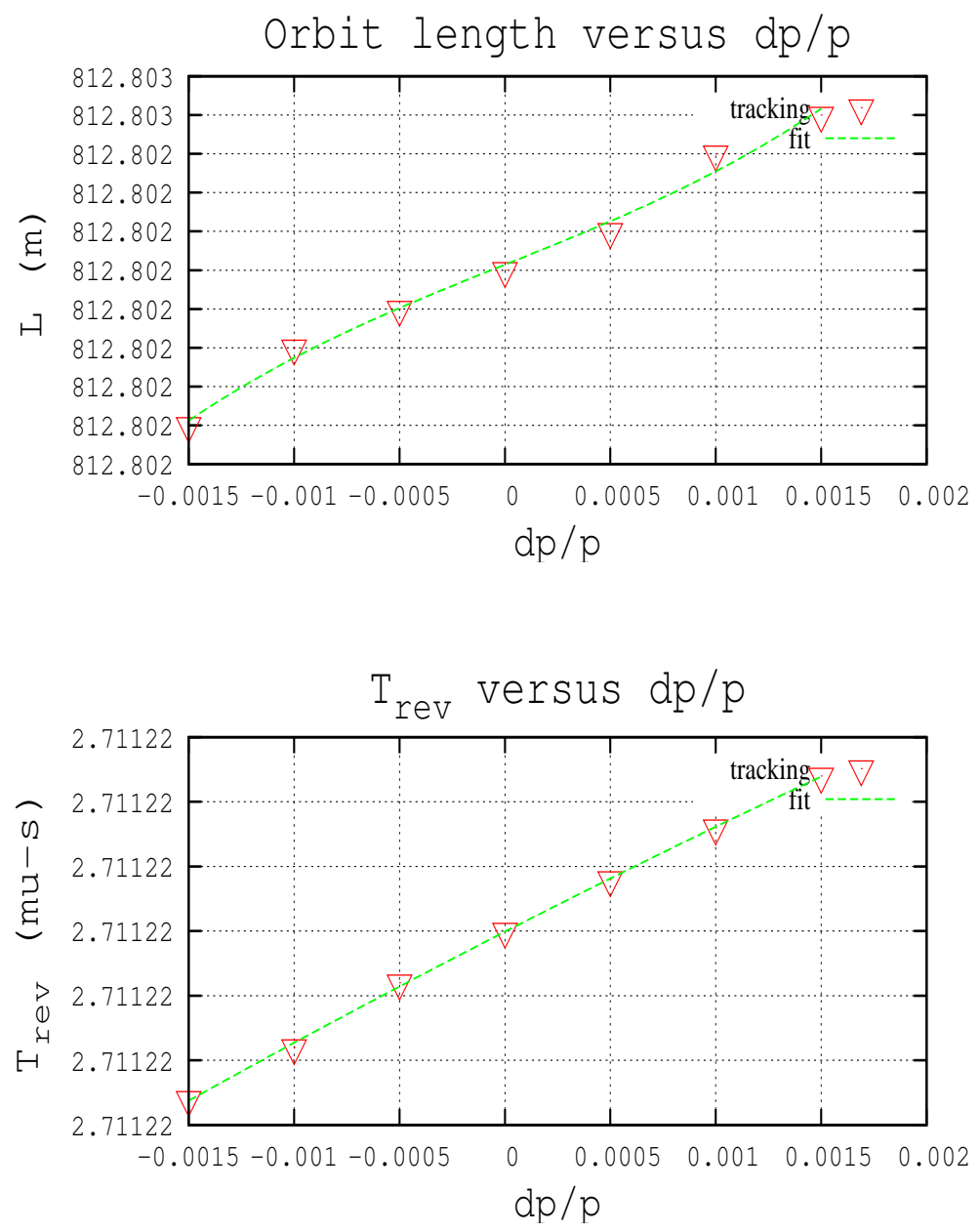

Figure 10: Top : momentum dependence of orbit length. Bottom : revolution period.

$L_{0}=812.80219, \gamma_{t r}=61.025505, \alpha_{0}=2.68520380 E-04, \alpha_{1}=-9.00838813 E-07, \alpha_{2}=27.340271$ $T_{0}=2.7112164, \eta=-3.08261689 E-04, \eta_{1}=8.78189132 E-03, \eta_{2}=1.05943894 E-02$

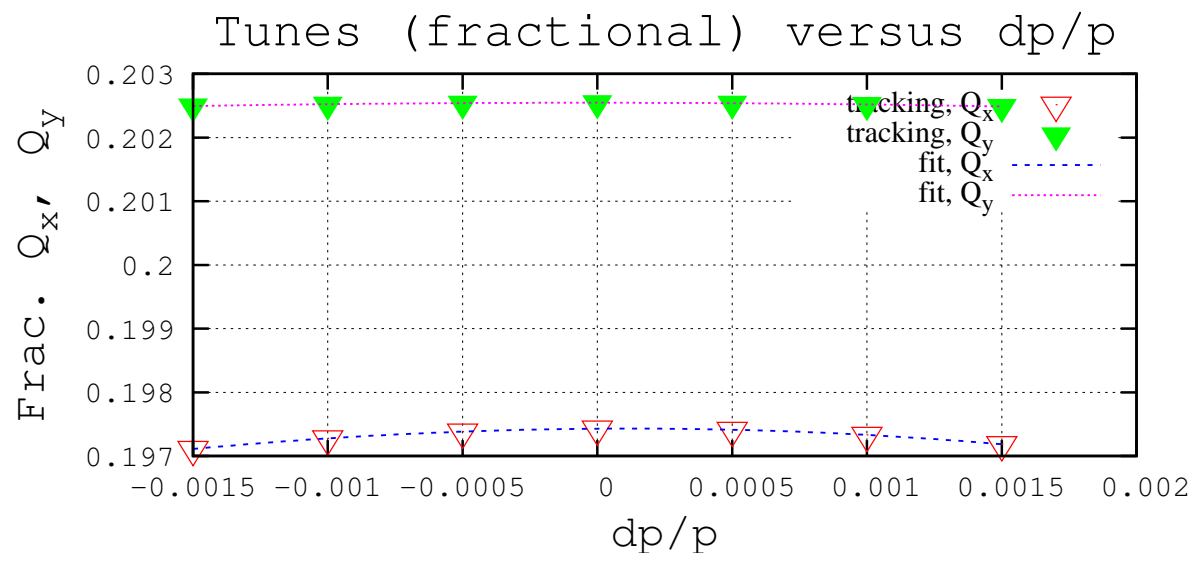

Figure 11: Momentum dependence of tunes, and matching polynomials $Q(\delta)=Q_{0}+Q^{\prime} \delta+Q^{\prime \prime} \delta^{2} Q^{\prime \prime \prime} \delta^{3}$.

Horizontal : $Q_{x}=0.19742838, Q_{x}^{\prime}=2.98930947 E-02, Q_{x}^{\prime \prime}=-125.03857$

Vertical : $Q_{y}=0.20254529, Q_{y}^{\prime}=-1.69357145 E-03, Q_{y}^{\prime \prime}=-24.214762$ 


\section{E Typical Zgoubi i/o files}

The two sections below show typical input ("zgoubi.dat") and output ("zgoubi.res") zgoubi files, as manipulated in the present benchmarking. There may be additional output files from Zgoubi, if requested, as for instance "zgoubi.fai", a record of local particle and spin coordinates, turn after turn, "zgoubi.plt", a record of coordinates and fields inside optical elements,

\section{E.1 Input data file zgoubi.dat}

Zgoubi execution requires an input data file, zgoubi.dat. That data file contains the ring optics sequence, a sequence of dipoles, quadrupoles, sextupoles and other drifts. Special instructions appear, in addition, usually at the top or at the bottom of the stack, they are executed in sequence with the transport of the particles through the optics sequence.

Excerpts from zgoubi.dat (top and bottom of the file) are shown hereafter.

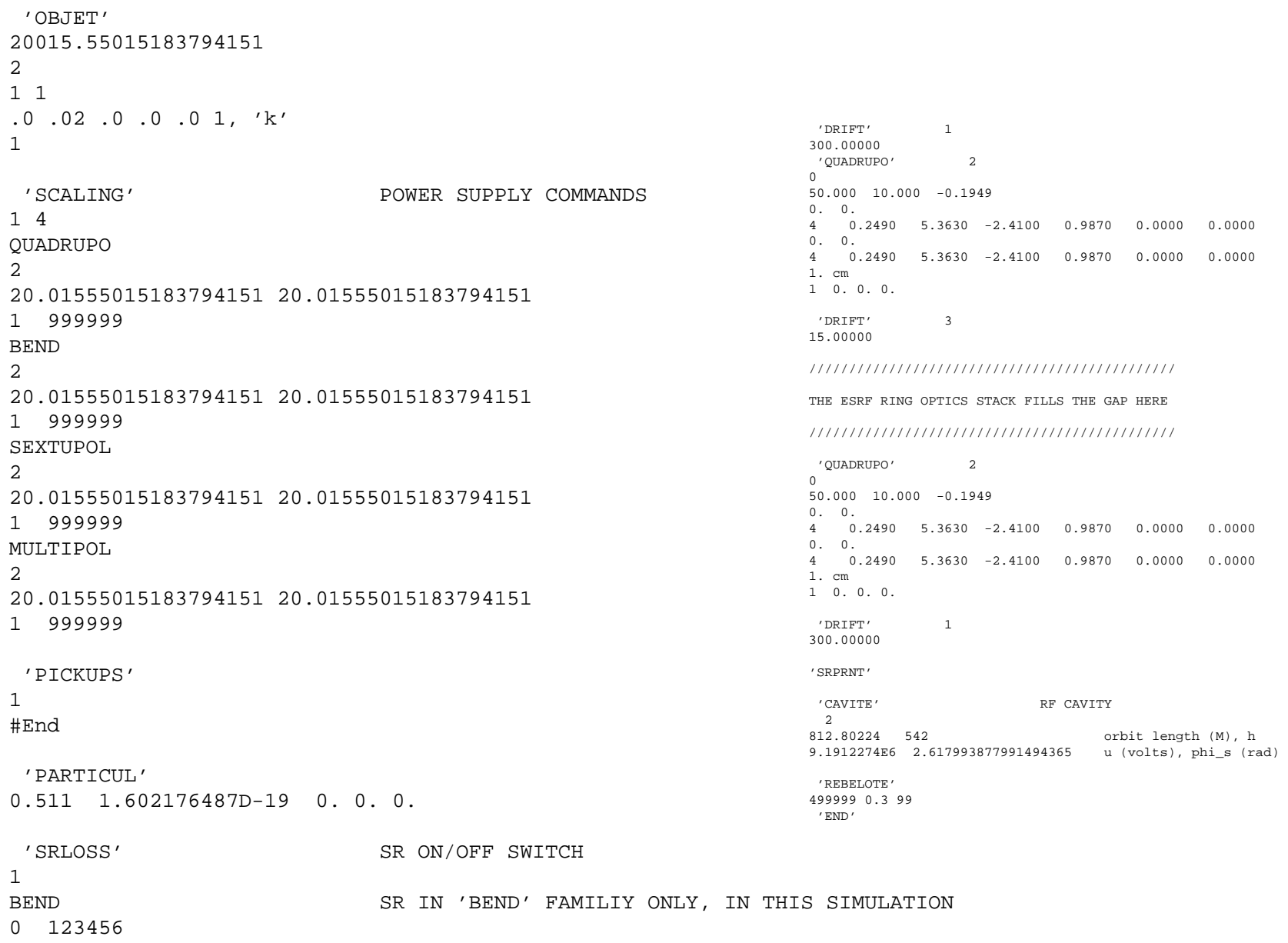

\section{E.2 Output data file zgoubi.res}

When executing, zgoubi produces, a minima, the output file zgoubi.res. Excerpts are displayed and briefly commented, here.

- Switching on SR Monte Carlo simulation :

6 Keyword, label(s) : SRLOSS

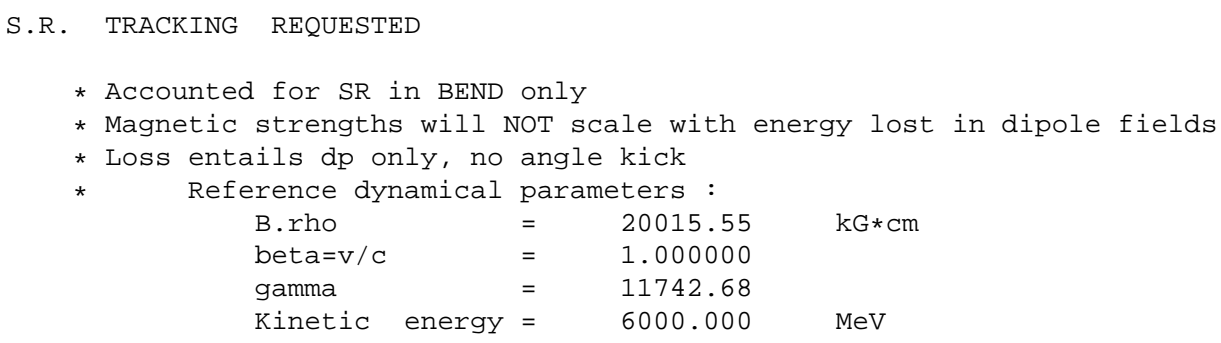


- Each time "BEND" is met, SR statistics data are printed out. The following is at the last bend in the ring, after 100 turns, 100 particles, yielding a summed $459561.37 \mathrm{keV} /$ particle energy loss or $4.5956 \mathrm{MeV} / \mathrm{turn}$, the value quoted in Table 1. Also quoted in Table 1, the present $19.204983 \mathrm{keV}$ critical energy, and 77712.607, the total number of average photons radiated by the 100 particles, after 100 turns.

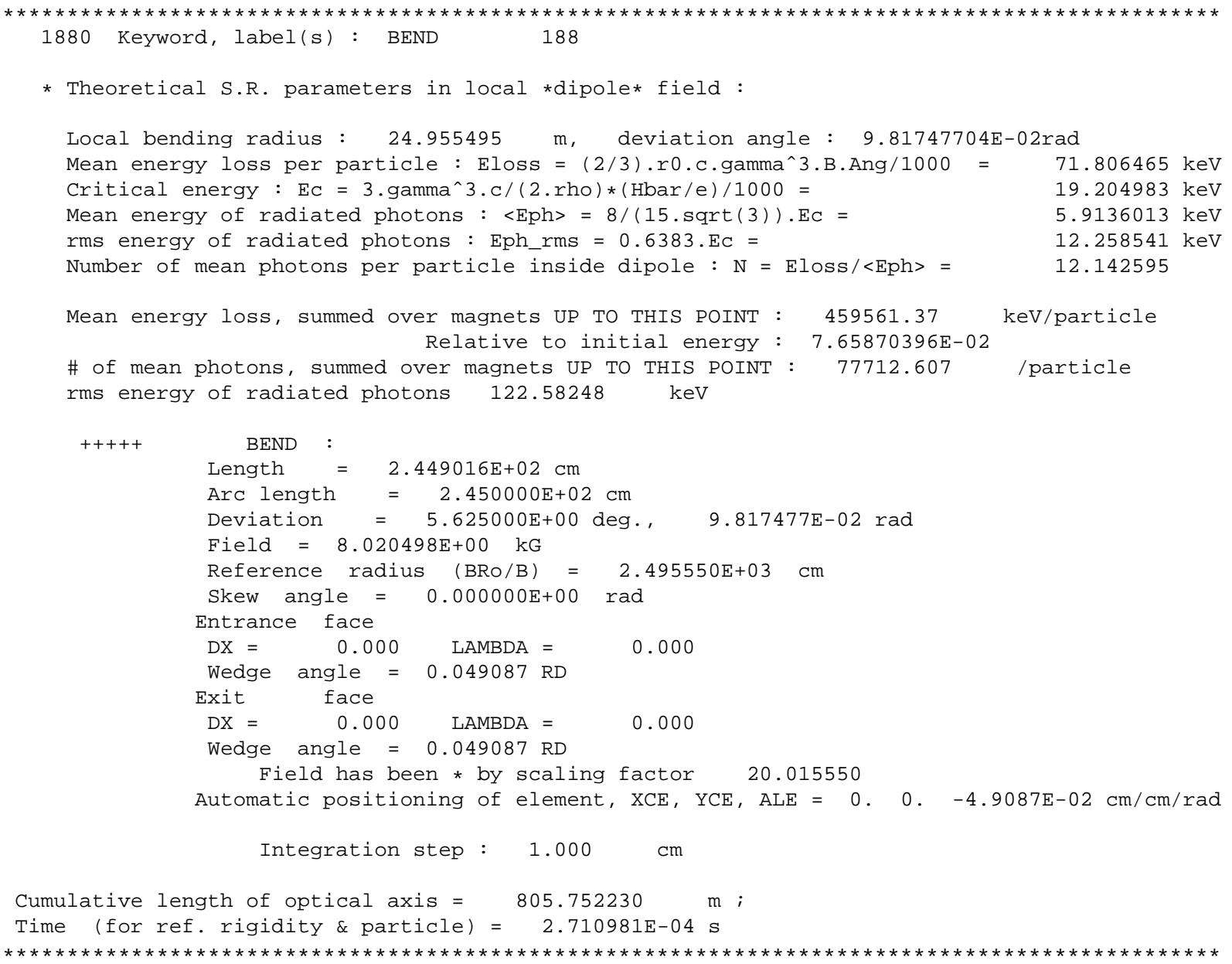

- When "REBELOTE" is met, i.e., at the end of each pass through the optics sequence, the program execution pointer is sent back to the top of the zgoubi.dat stack.

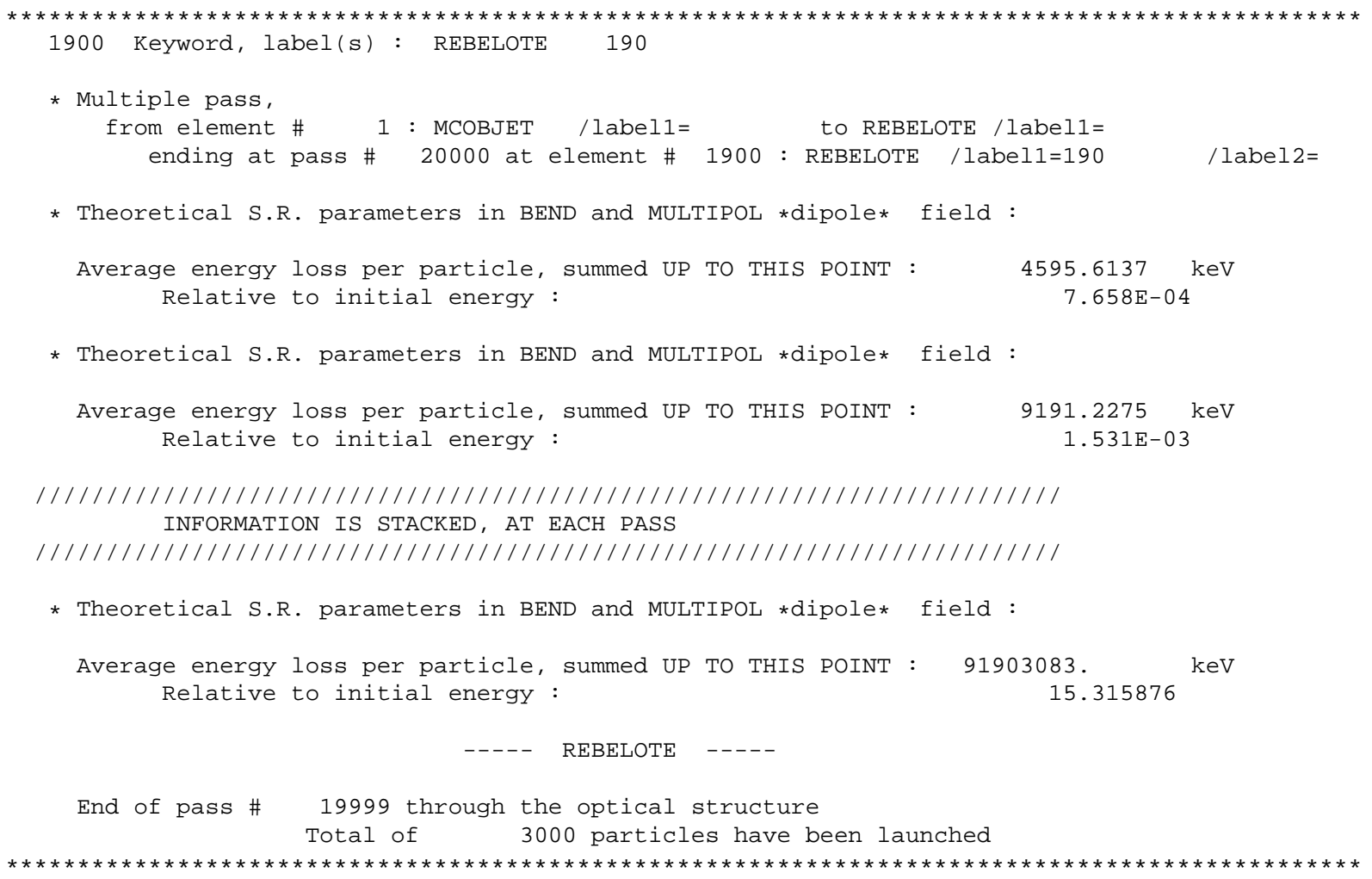


- SR information output using "SRPRNT"

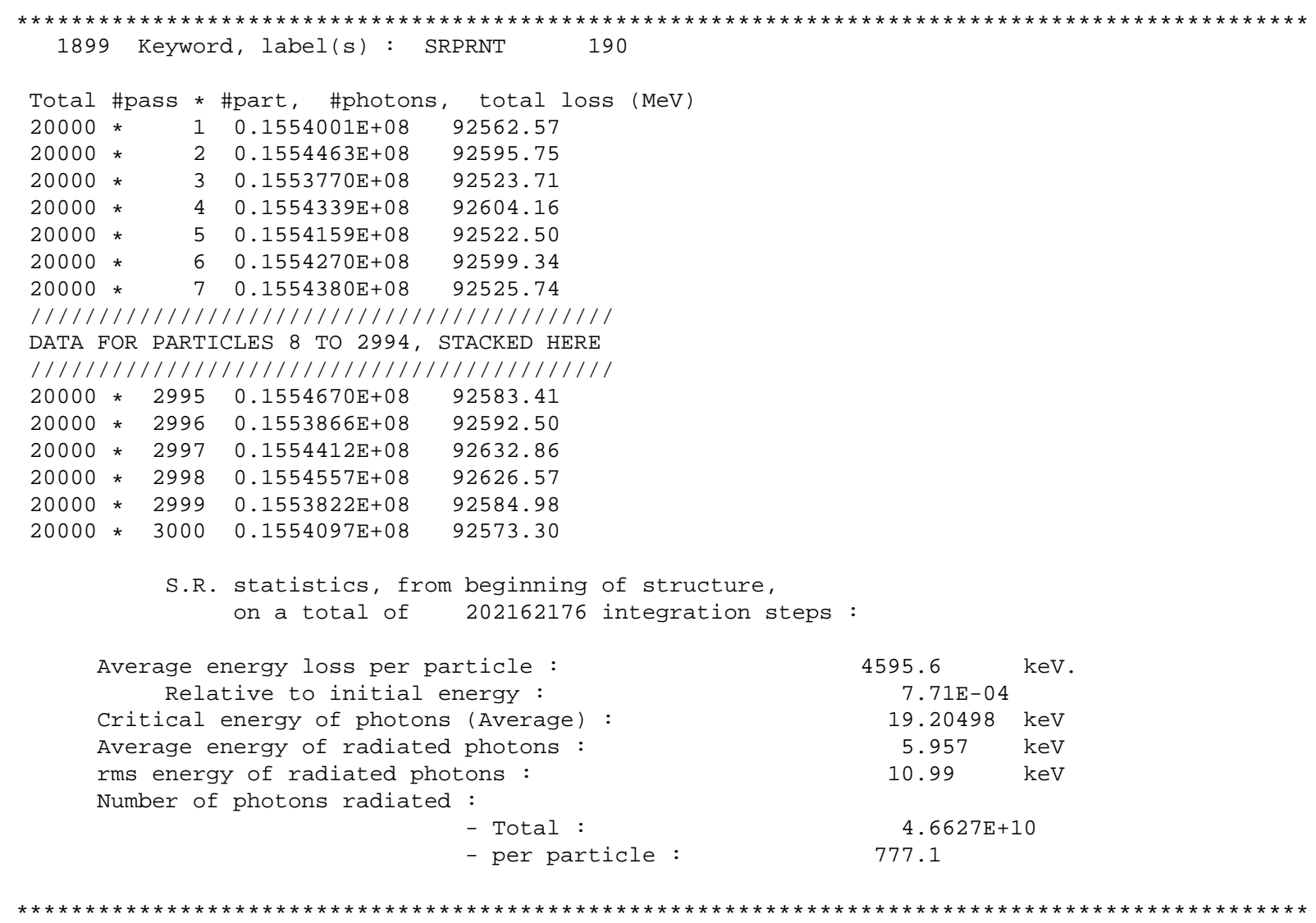

\section{F Tracking, typical data}

\section{F.1 Longitudinal motion}

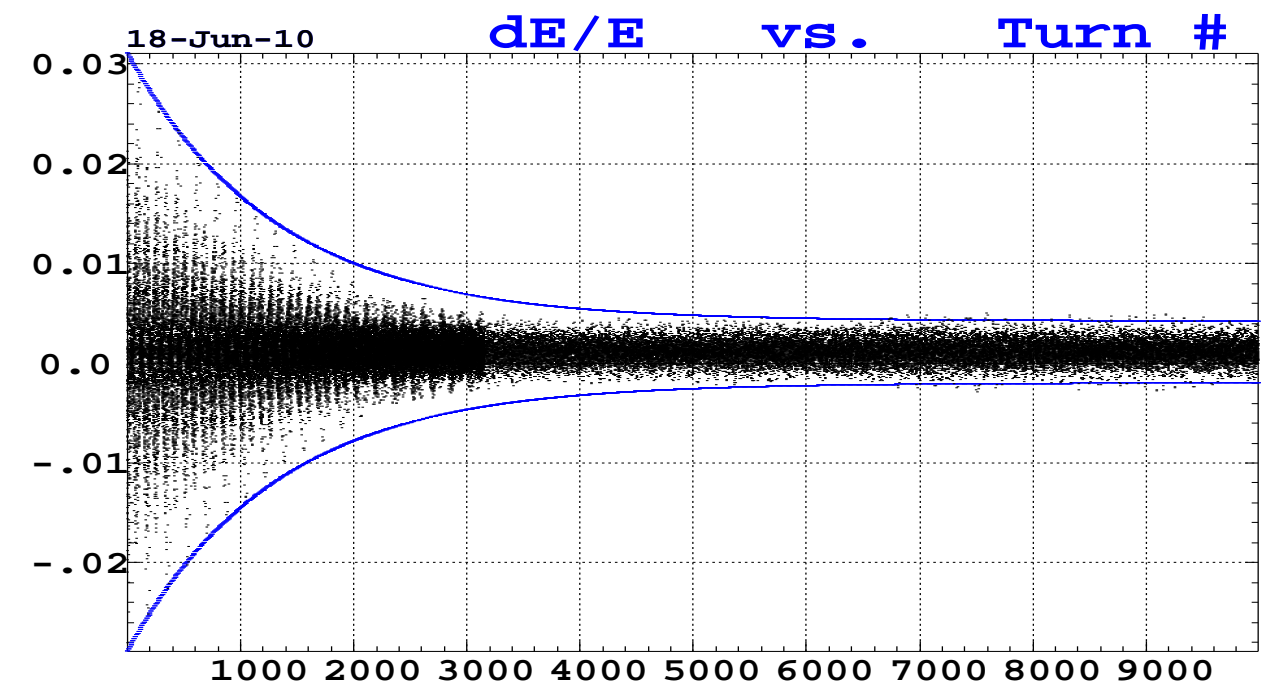

Damping of the longitudinal motion : 1000 particles are launched, with Gaussian energy density with width about 10 times the natural $\sigma_{\frac{d E}{E}}$. A fit of the last few 1000 turns yields :

- average $\mathrm{dE} / \mathrm{E} \approx 0.3810^{-3}$,

- rms spread $\sigma_{\frac{d E}{E}} \approx 1.02310^{-3} \mathrm{~m}$ (theoretical is $1.02810^{-3}$ ),

- average RF phase is $2.6136 \mathrm{rad}$,

- rms phase $\approx 2.284710^{-2}$, hence bunch length $2.2847110^{-2} \times c /\left(2 \pi f r e q_{R F}\right)=9.40 \mathrm{~mm}$ (theoretical is $\left.9.30 \mathrm{~mm}\right)$. 


\section{F.2 Horizontal motion}

1000 particles are launched, with Gaussian 2-D horizontal density and CE-Surface $\epsilon_{x, \text { initial }} / \pi=300 \mathrm{~nm}$, about 44 times the natural emittance (Fig. 12), with zero vertical invariants and zero starting momentum spread.

Initial and final horizontal phase spaces are given in Figs. 12, 13 respectively.

Damping of the horizontal motion at $6 \mathrm{GeV}$ is shown in Fig. 14. The envelopes $\sigma_{x}= \pm \sqrt{\beta_{x} \epsilon_{x} / \pi}$ (solid lines) follow from Eq. 8 with $\epsilon_{x, \text { equil }} / \pi=6.8310^{-9} \mathrm{~m}$.rad the $r m s$ beam emittance and with damping time $\tau_{x}=n_{x}$ [turns] $\times T_{\text {rev }} \approx 7.08 \mathrm{~ms}\left(n_{x}=2610\right.$, twice the emittance damping time, see Tab. 5, page 9).

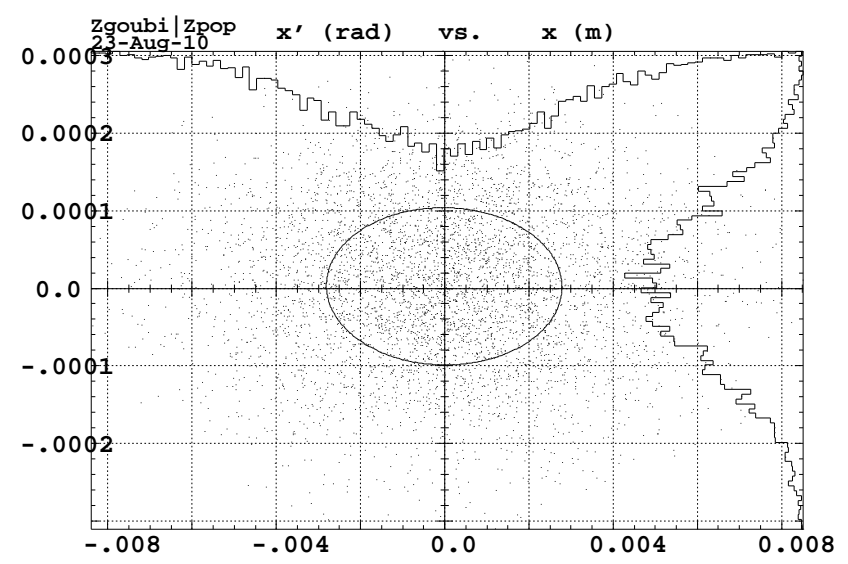

Figure 12: Starting horizontal phase-space.

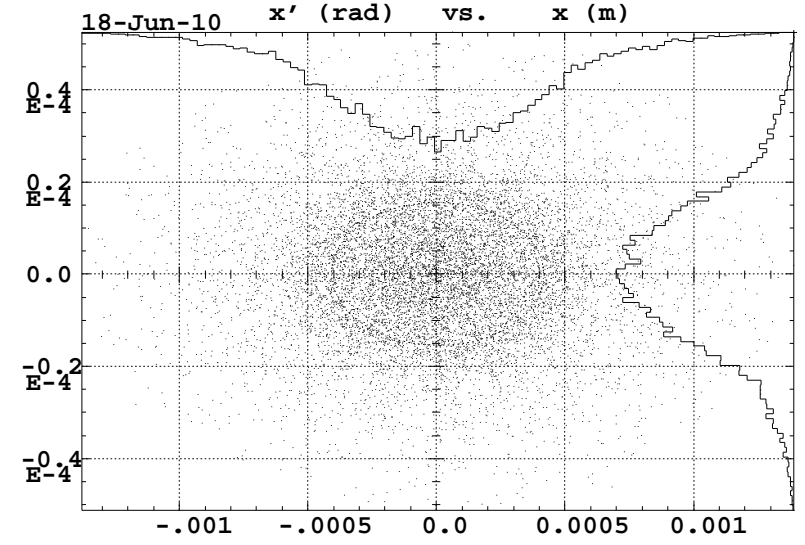

Figure 13: Final horizontal phase-space, a projection of turns number 9500 to 10000 . The asymptotic $r m s$ emittance is $\epsilon_{x} / \pi \approx 6.4 \mathrm{~nm}$, close to the expected, theoretical $6.8 \mathrm{~nm}$.

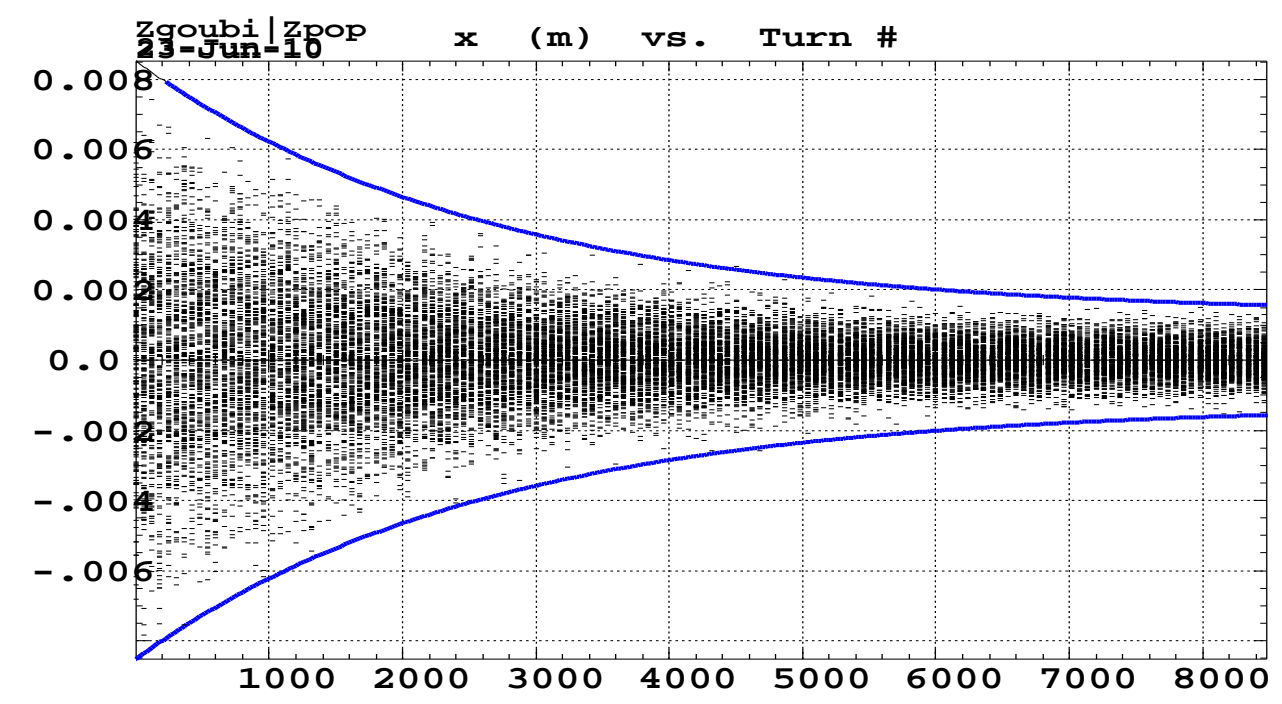

Figure 14: Damping of the horizontal motion, $6 \mathrm{GeV}$, with exponential decay envelopes.

\section{F.3 Vertical motion}

Sample outcomes similar to the previous ones are displayed in Fig. 3 p. 8. 


\section{F.4 Coupled optics}

Coupling is obtained by introducing a skew quad at the azimuth $\mathrm{s}=0$ (Fig. 2) in the lattice. Tracking of a (few) hundred particles, over 20000 turns, is performed with various coupling strengths $b_{s} / B \rho$ with $b_{s}$ the quadrupole field at pole tip.

The following figures display typical turn dependence of the transverse excursions, $\mathrm{x}$, and $\mathrm{y}$, and their evolution with the coupling strength (horizontal motion is damped, vertical motion is growing). The short tables display typical numerical data drawn from these tracking trials. In presence of strong $b_{s}$ (first cases), the horizontal motion damps towards a larger equilibrium emittance than in the case of a weaker $b_{s}$ (last cases). This type of data yielded the results displayed in Fig. 7.

\section{$6 \mathrm{GeV}$}

- $b_{s}=0.0710^{-3}$ T.m ;

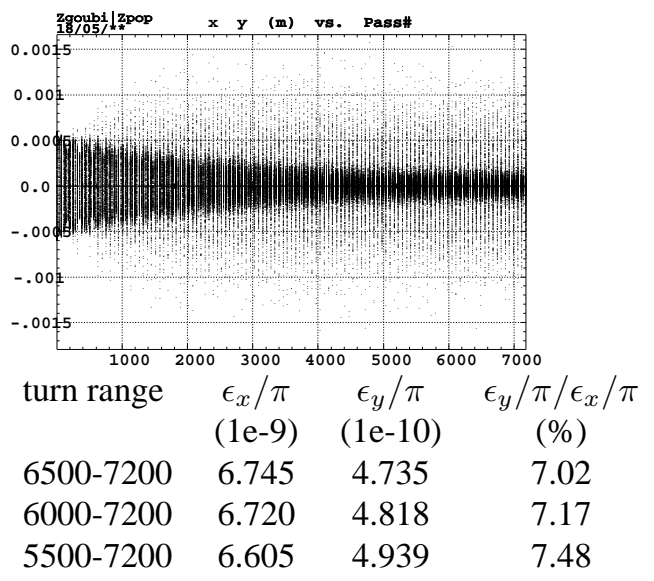

- $b_{s}=0.04510^{-3}$ T.m

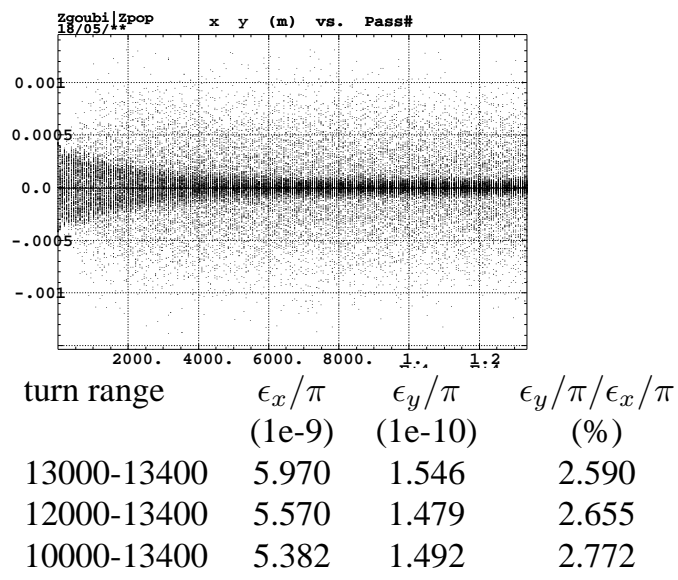

- $b_{s}=0.01 e 310^{-3}$ T.m

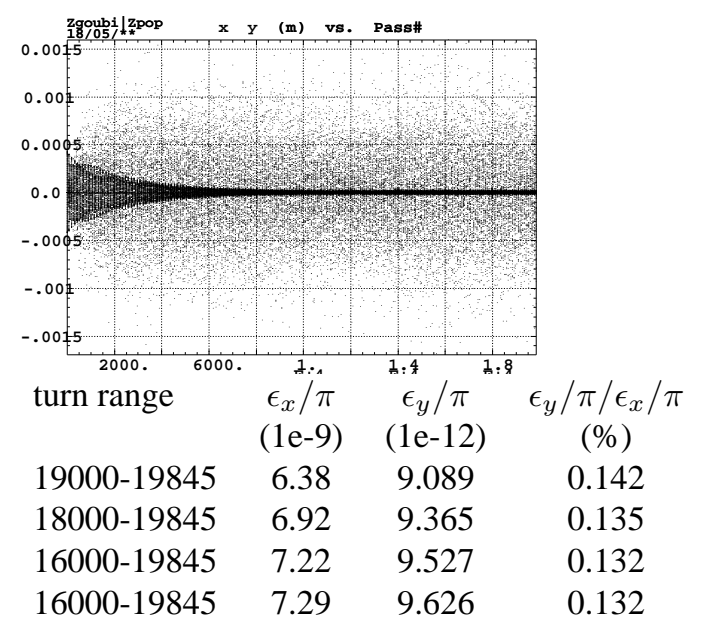

Final $\left(\mathrm{x}, \mathrm{x}^{\prime}\right)$

Final $(y, y$ ')

after 20000 turns.
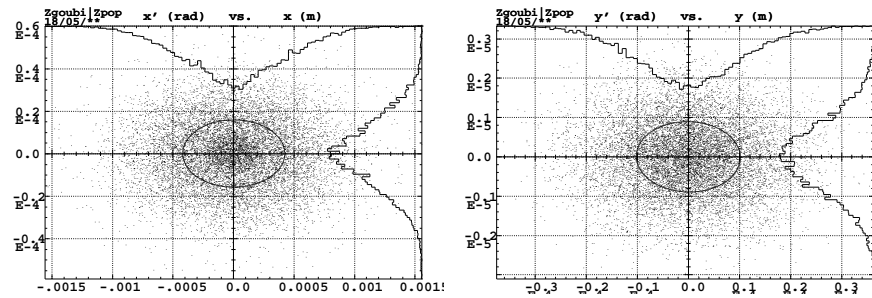


\section{References}

[1] The ray-tracing code Zgoubi, F. Méot, Report BNL-98726-2012-IR, Oct. 2012, http://www.scienceaccelerator.gov/dsa/result-list/fullRecord:zgoubi/

Source files and examples are available at http://sourceforge.net/projects/zgoubi/

[2] Numerical tools for the simulation of synchrotron radiation loss and induced dynamical effects in high energy transport lines, F. Méot, J. Payet, int. rep. DAPNIA/SEA-00-01, CEA/DSM Saclay (July 2000).

[3] A new dynamics code DYNAC for electrons, protons and heavy ions in LINACS with long accelerating elements, P. Lapostolle, F. Méot, S. Valero, 1990 LINAC Conf., Albuquerque, NM, USA ; report SATURNE/GT/90-04, CEA/DSM Saclay (1990).

[4] Electron Laboratory For Europe, "Blue Book" Accelerator Technical Proposal, CNRS-IN2P3 editor, Institut des Sciences Nucléaires, Grenoble, France (1994).

[5] Longitudinal emittance growth induced by SR in the arcs of a CEBAF like machine, G. Leleux, P. Nghiem, A. Tkatchenko, Report DSM/SATURNE LNS/GT/90-08, CEA Saclay (1990).

[6] Synchrotron radiation interferences between short dipoles at LEP, C. Bovet, A. Burns, F. Méot, M. Placidi, E. Rossa, J. De Vries, rep. CERN SL/97-59 (BI), Nov. 1997.

A theory of low frequency, far-field synchrotron radiation, F. Méot, Particle Accelerators, Vol. 62 (1999), pp. 215-239.

[7] LHC proton beam diagnostics using synchrotron radiation, L. Ponce, R. Jung, F. Méot, Yellow Report CERN-2004-007.

[8] A numerical method for combined spin tracking and ray tracing of charged particles, F. Méot, NIM A 313 (1992) 492-500.

[9] Dynamics studies regarding the super-B lattice, status report, F. Méot, Elba Super-B Collaboration Workshop, Italy, June 2010 ; Lattice design and study tools regarding the super-B project, F. Méot, N. Monseu, Procs. IPAC10, Kyoto, Japan ;

Spin Dynamics Tools Development and Study, Regarding the Super-B Project, N. Monseu, et al., http://accelconf.web.cern.ch/AccelConf/IPAC2011/papers/thpz001.pdf.

[10] High luminosity electron-hadron collider eRHIC, V. Ptitsyn et al., http://accelconf.web.cern.ch/AccelConf/IPAC2011/papers/thpz019.pdf.

[11] Synchrotron radiation, G Leleux, SATURNE Lab., CEA Saclay, 1989, unpublished.

[12] BETA users' guide, J. Payet, DSM/IRFU/SACM, CEA Saclay.

[13] Betatron coupling with radiation, G. Guignard, CERN Accelerator School, Yellow Rep. CERN 87-03, 21 Apr. 1987.

[14] Spin code benchmarking at RHIC, F. Méot et al., Procs. Particle Accelerator Conference 2011, New York, USA, 2011 ; Polarization transmission at RHIC, numerical simulations, F. Méot et al., Procs. IPAC2012, New Orleans, Louisiana, USA. 